\title{
Tipos de demostraciones
}

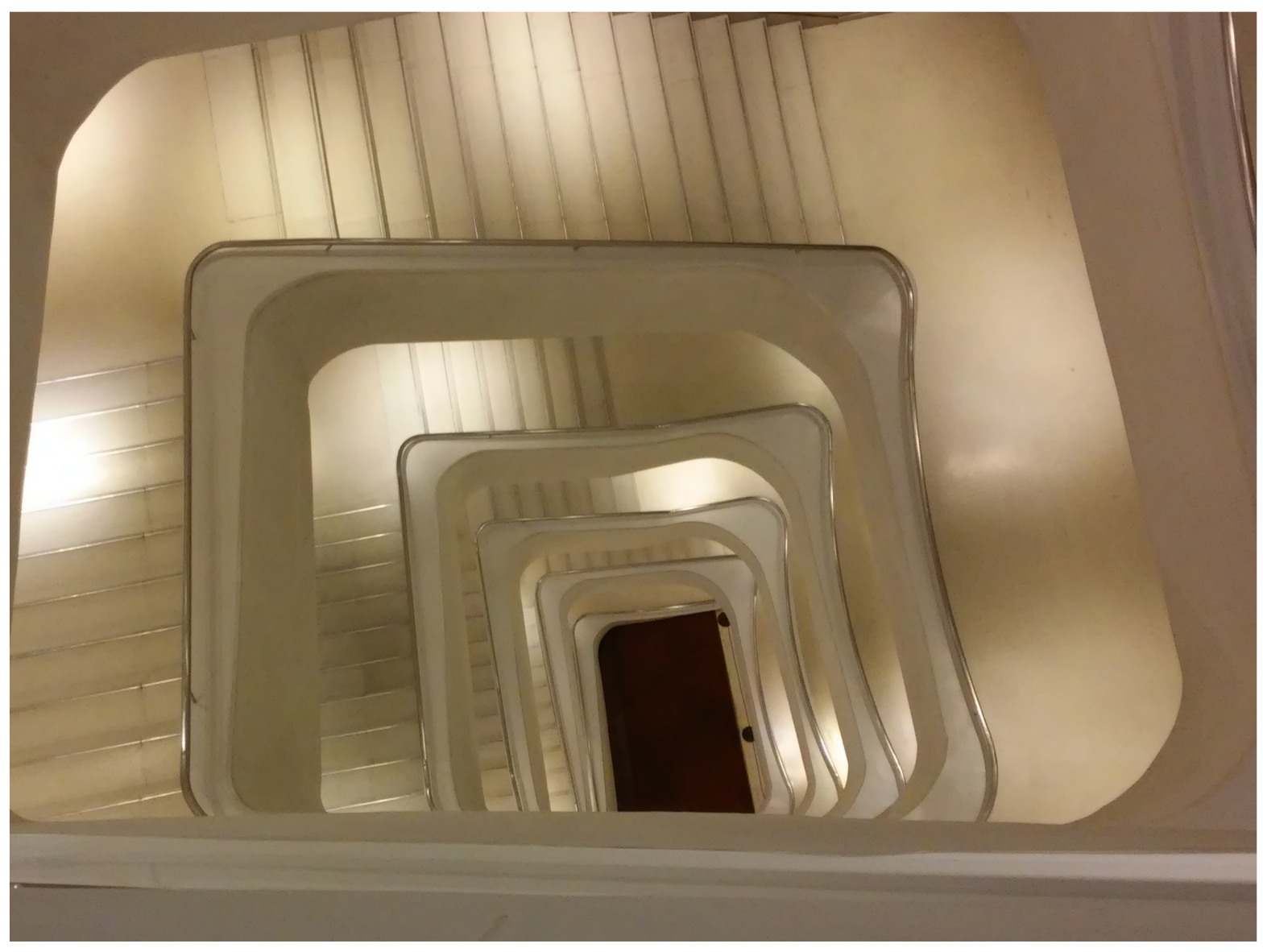

Ma Ángeles Rincón Ortega Emilio Letón Molina

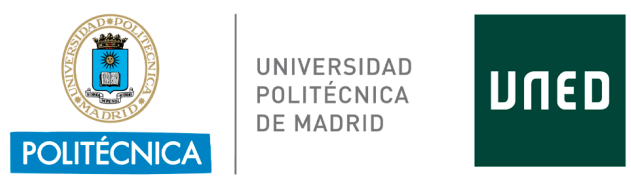





\section{Página legal}

\section{TIPOS DE DEMOSTRACIONES}

@ $\mathrm{M}^{\mathrm{a}}$ Ángeles Rincón Ortega. Profesora del departamento de Matemática Aplicada a la Ingeniería Industrial de la Universidad Politécnica de Madrid (UPM).

@a Emilio Letón Molina. Profesor del departamento de Inteligencia Artificial de la Universidad Nacional de Educación a Distancia (UNED).

www.minixmodular.ia.uned.es

Madrid, 2021

Obra licenciada bajo licencia Creative Commons

Reconocimiento-NoComercial-CompartirIgual 3.0 España:

http://creativecommons.org/licenses/by-nc-sa/3.0/es/legalcode.es 



\section{Prefacio}

Este material está diseñado con el paradigma del grupo de innovación docente miniXmodular de generar material en formato mini y modular, según se puede ver en la página web www.minixmodular.ia.uned.es, donde se introduce, entre otros, el concepto de mini-libro electrónico modular. Este mini-libro pretende ayudar a entender los tipos de demostraciones que aparecen de forma habitual en el campo de las Matemáticas, buscando los siguientes objetivos:

- Distinguir las demostraciones directas de las demostraciones a la contra.

- Interpretar las condiciones necesarias y suficientes.

- Estudiar las demostraciones por reducción al absurdo y por marcha hacia atrás.

- Manejar el principio de inducción en su versión simple y completa.

- Manejar el principio de buena ordenación y de descenso infinito.

En Matemáticas es habitual hacerse preguntas. Decía el sabio que las preguntas son más importantes que las respuestas. Es bueno cuestionarse:

1. ¿Si pasa esto, pasa lo otro?

2. O es al revés, ¿si pasa lo otro, pasa esto?

En Matemáticas a las afirmaciones del tipo "Si pasa esto, entonces pasa lo otro" se las representa por " $A \Rightarrow B$ " y se lee como "si $A$ entonces $B$ " o " $A$ implica $B$ " (implicación hacia la derecha). En dicha expresión, $A$ es la hipótesis y $B$ es la tesis. Es conveniente observar que puede ocurrir que $A \Rightarrow B$ pero que no se dé el recíproco, es decir que $A \nLeftarrow B$, que se lee como " $B$ no implica $A$ " o que " $A$ no es implicado por $B$ ".

En algunos casos puede ocurrir, sin embargo, que $A \Rightarrow B$ y a la vez $A \Leftarrow B$ (implicación hacia la izquierda), en cuyo caso se escribe $A \Leftrightarrow B$ y se lee como " $A$ equivale a $B$ ". A veces puede ocurrir que $A \nRightarrow B$ y que $B \nRightarrow A$.

Para demostrar que algo se cumple en matemáticas, no basta con demostrarlo para unos pocos casos, ni siquiera para muchísimos casos, sino que hay que demostralo para todos los casos posibles (es muy importante ver ahora el mini-vídeo '¿Cuántos casos?', Sin embargo, para demostrar que algo no se cumple basta encontrar un ejemplo de ello, al cual se le llama "contraejemplo".

Existen distintas formas de demostración, siendo los principales métodos: el método directo y a la contra (ver el mini-capítulo "Directas y contras"), el método de reducción al absurdo y marcha hacia atrás (ver mini-capítulo "Supongamos que"), el método de inducción simple e inducción completa (ver mini-capítulo "Inducción") y el principio de buena ordenación y de descenso infinito (ver mini-capítulo "Primer elemento").

A la hora de plantear cualquier demostración es importante distinguir los conceptos de hipótesis y tesis y de condición necesaria y suficiente (ver mini-capítulo "Necesaria y suficiente").

Las demostraciones en este mini-libro, sea cual sea el método que se emplee para ello, se acaban con un $\square$ (a veces, en la literatura también se usa "c.q.d" (como queríamos demostrar) o "q.e.d" (quod erat demostrandum).

En este mini-libro se manejan distintos conjuntos de números:

- Los naturales, que se denotan por $\mathbb{N}$, y que están constituidos por $\{1,2,3, \ldots\}$.

- Los enteros, que se denotan por $\mathbb{Z}$, y que están constituidos por $\{\ldots,-3,-2,-1,0,1,2,3, \ldots\}$.

- Los racionales, que se denotan por $\mathbb{Q}$, y que están constituidos por $\left\{\frac{m}{n}, m \in \mathbb{Z}, n \in \mathbb{Z}-\{0\}\right\}$. 
- Los reales, que se denotan por $\mathbb{R}$, y que de forma intuitiva están constituidos por los puntos de la recta del eje horizontal de coordenadas.

Adicionalmente, se presupone que el lector de este mini-libro sabe operar con los números naturales, enteros y racionales, y que conoce los conceptos de divisor y múltiplo, las igualdades básicas algebraicas (cuadrado de una suma y diferencia de cuadrados) y operar con desigualdades. Es interesante ver ahora el mini-vídeo 'Demostración falsa I'.

Así mismo, en este mini-libro aparece el conjunto de números primos (un número natural distinto de uno es primo si solo tiene por divisores al 1 y a sí mismo) y número compuesto (un número natural que no es primo). Por convenio se dice que el 1 no es ni primo ni compuesto, es la unidad. Es interesante ver ahora el mini-vídeo 'Números primos'.

Por último hay que señalar que en este mini-libro, los mini-vídeos que se mencionan en él siguen el concepto de mini-vídeos docentes modulares (MDM). Cada MDM tiene asociado sus trasparencias minimalistas (TM) en formato PDF alojados junto a su MDM. Se aconseja imprimir dichas TM e ir completándolas mientras se ven los MDM correspondientes. El concepto MDM se explica en el mini-vídeo 'Características de un MDM', Así mismo, hay un mini-capítulo de autoevaluación de conocimientos requeridos y otro de referencias de interés (ver [1], [2], 3] y [4).

Es importante indicar que este mini-libro está "animado "a través del hashtag \#tdmXm 


\section{Índice general}

Autoevaluación de conocimientos requeridos 1

1. Directas y contras 3

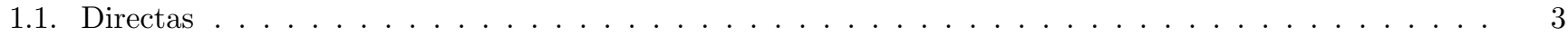

1.2. Directas globales . . . . . . . . . . . . . . . . . . . . . . . . . . 6

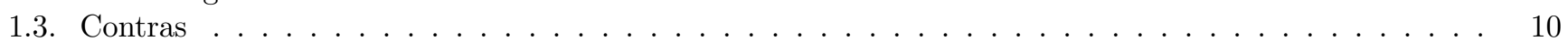

2. Necesaria y suficiente 13

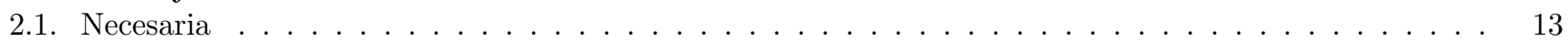

2.2. Suficiente $\ldots \ldots \ldots \ldots \ldots$

3. Supongamos que 21

3.1. Reducción al absurdo . . . . . . . . . . . . . . . . . . . . . . . . . . . . . . . 21

3.2. Marcha hacia atrás . . . . . . . . . . . . . . . . . . . . . . . . . . 23

4. Inducción 2

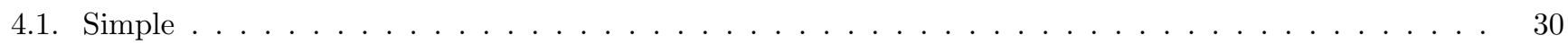

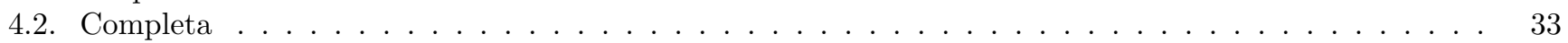

5. Primer elemento $\mathbf{3 7}$

5.1. Principio de buena ordenación . . . . . . . . . . . . . . . . . . . . . . . . . . . . . 37

5.2. Principio de descenso infinito . . . . . . . . . . . . . . . . . . . . . . . . . 41

6. Autoevaluación de contenidos 43

6.1. Directas y contras . . . . . . . . . . . . . . . . . . . . . . . . . . . . . 43

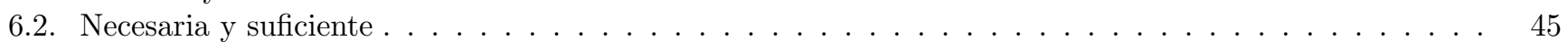

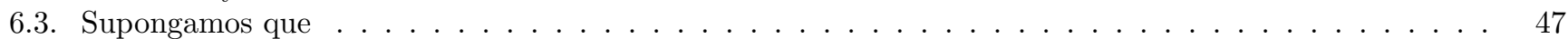

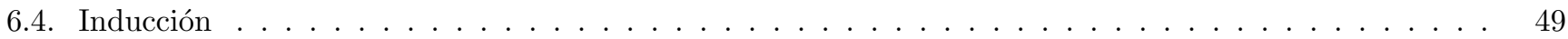

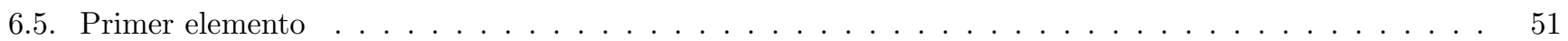

6.6. Respuestas correctas . . . . . . . . . . . . . . . . . . . . . . . . . . 54

\begin{tabular}{ll} 
7. Glosario & 55 \\
\hline
\end{tabular} 



\section{Autoevaluación de conocimientos}

\section{requeridos}

- A continuación se presenta una autoevaluación que pretende evaluar el nivel de conocimientos básicos matemáticos del lector. Sería recomendable que se obtuviese ocho o más respuestas acertadas en esta autoevaluación.

1. 63 es un número primo.

a) Verdadero.

b) Falso.

SOLUCIÓN: La respuesta correcta es la $b$ ).

2. 17 es un número compuesto.
a) Verdadero.
b) Falso.

SOLUCIÓN: La respuesta correcta es la $b$ ).

3. 48 es un múltiplo de 12 .
a) Verdadero.
b) Falso.

SOLUCIÓN: La respuesta correcta es la $a$ ).

4. $(a-b)^{2}=a^{2}-b^{2}-2 a b$.
a) Verdadero.
b) Falso. 
SOLUCIÓN: La respuesta correcta es la $b$ ).

5. Solo hay un número primo que a la vez es par.

a) Verdadero.

b) Falso.

SOLUCIÓN: La respuesta correcta es la $a$ ).

6. $(2 a)(2 a)=4 a^{2}$.

a) Verdadero.

b) Falso.

SOLUCIÓN: La respuesta correcta es la $a$ ).

7. $\frac{1}{2}+\frac{1}{2^{2}}=\frac{3}{4}$.

a) Verdadero.

b) Falso.

SOLUCIÓN: La respuesta correcta es la $a$ ).

8. $\frac{\frac{2^{2}-1}{2^{2}}}{\frac{1}{2^{2}}}=\frac{2^{2}-1}{2}$.

a) Verdadero.

b) Falso.

SOLUCIÓN: La respuesta correcta es la $b$ ).

9. $(-4)(+3)=-12$.
a) Verdadero.
b) Falso.

SOLUCIÓN: La respuesta correcta es la $a$ ).

10. $(n+1)(n-1)=n^{2}-1$.
a) Verdadero.
b) Falso.

SOLUCIÓN: La respuesta correcta es la $a$ ). 


\section{Capítulo 1}

\section{Directas y contras}

\section{Objetivos}

- Empezar a conocer ejemplos concretos de demostraciones directas y contras.

- Saber la relación entre directas y contras.

- Comenzar a plantearse posibles hipótesis y tesis.

En este mini-capítulo se describen dos formas de demostrar afirmaciones del tipo $A \Rightarrow B$, demostraciones directas y demostraciones a la contra (contrarrecíprocas). En las primeras se considera además la posibilidad de que sean globales para indicar que la hipótesis es global ("todo lo que se sabe de un tema o contexto"). En la sección 1.1 se explica lo relativo a las demostraciones directas, en la sección 1.2 las demostraciones directas globales y en la sección 1.3 las demostraciones a la contra.

Señalar que habrá ejemplos en los que se da $A \Rightarrow B$, pero $B \nRightarrow A$ y ejemplos en los que $A \Rightarrow B$ y $B \Rightarrow A$. Es importante observar que puede haber casos en los que $A \nRightarrow B$ y $B \nRightarrow A$. Por ejemplo primo $\nRightarrow$ capicúa (basta tomar el número 17 que es primo y no es capicúa) y que capicúa $\nRightarrow$ primo (se ve con el $121=11^{2}$ que es capicúa y no es primo).

Es conveniente ver el MDM "Directas y contras" antes de seguir leyendo este mini-capítulo.

\subsection{Directas}

En las demostraciones directas para demostrar que $A \Rightarrow B$ se parte de la hipótesis $A$ y realizando razonamientos "directos" se llega a la tesis $B$. 


\section{Ejemplos}

1. Demostrar que el producto de dos números naturales pares $a$ y $b$ siempre es par. Esta afirmación se representa por

$a$ y $b$ son dos números naturales pares $\Rightarrow a \cdot b$ es par.

\section{SOLUCIÓN:}

Se tiene que demostar la implicación " $\Rightarrow$ " y se hará utilizando razonamientos directos. Este esquema de demostración se escribe en este mini-libro como " $\Rightarrow$ " (Directa).

" $\Rightarrow "($ Directa)

Se supone que $a$ y $b$ son pares, es decir que son múltiplos de 2, por lo que de forma genérica se pueden escribir como $a=\dot{2}$ y $b=\dot{2}$, donde el punto superior indica "múltiplo de". Por tanto, $a=2 k_{1}$ y $b=2 k_{2}$, con $k_{1}, k_{2} \in \mathbb{N}$, por lo que

$$
a \cdot b=\left(2 k_{1}\right)\left(2 k_{2}\right) \stackrel{\text { Asociativa }}{=} 2 k_{1} \cdot 2 k_{2} \stackrel{\text { Conmutativa }}{=} 2 \cdot 2 k_{1} k_{2} \stackrel{\text { Asociativa }}{=} 2\left(2 k_{1} k_{2}\right)=2 k_{3}=\dot{2}
$$

ya que $k_{3}=2 k_{1} k_{2} \in \mathbb{N}$.

Obs. 1: Una vez terminada la demostración es conveniente preguntarse por si será cierto el recíproco $A \Leftarrow B$, es decir, por si será cierta la afirmación " $a$ y $b$ son dos números naturales pares $\Leftarrow a \cdot b$ es par", o lo que es lo mismo " $a \cdot b$ par $\Rightarrow a$ y $b$ pares". Se empieza a probar con algunos casos: $32=8 \cdot 4$ par $\Rightarrow 8$ par y 4 par, $12=6 \cdot 2$ par $\Rightarrow 6$ par y 2 par, $12=3 \cdot 4$ par $\nRightarrow 3$ par y 4 par, .... En este momento ya se tiene un caso para el que no se verifica la afirmación, se ha encontrado un contraejemplo, con lo que se puede afirmar que $A \nLeftarrow B$.

Obs. 2: Si se hubiera intentado hacer la demostración $A \Leftarrow B$, siguiendo el esquema de la demostración $A \Rightarrow B$, se tendría que se partiría de $a \cdot b$ par y habría que ver si se llega a que ambos son pares. Es decir, $a \cdot b=2 k \Rightarrow$ $a=2\left(k \cdot \frac{1}{b}\right)$ y que $b=2\left(k \cdot \frac{1}{a}\right)$. Pero ahora no se puede asegurar que $a$ y $b$ sean pares, porque no se tiene la seguridad de que $k \cdot \frac{1}{b} \in \mathbb{N}$ y de que $k \cdot \frac{1}{a} \in \mathbb{N}$ (el cociente de dos números naturales no tiene por qué ser natural), con lo que la demostración se queda atascada (lógico porque se sabía por el contraejemplo que no era cierta).

2. Sea $a \in \mathbb{N}$, demostrar que $a=\dot{12} \Leftrightarrow a=\dot{3}$ y $a=\dot{4}$.

\section{SOLUCIÓN:}

Esta equivalencia se demostrará en dos etapas. En la primera etapa se demuestra " $\Rightarrow$ ” usando una demostración directa y en la segunda etapa se demuestra “ $\Leftarrow "$ utilizando también una demostración directa.

" $\Rightarrow "$ (Directa) 
$a=12 \Rightarrow a=12 k=3 \cdot 4 k=\left\{\begin{array}{c}3 \cdot(4 k)=3 k^{\prime} \\ 4 \cdot(3 k)=4 k^{\prime \prime}\end{array}\right.$ con $k, k^{\prime}, k^{\prime \prime} \in \mathbb{N} \Rightarrow a$ es múltiplo de 3 y $a$ es múltiplo de 4. "६" (Directa)

$\left\{\begin{array}{l}a=\dot{3} \\ a=\dot{4}\end{array} \Rightarrow\left\{\begin{array}{l}a=3 k_{1} \\ a=4 k_{2}\end{array} \Rightarrow 3 k_{1}=4 k_{2} \Rightarrow\left\{\begin{array}{r}3 k_{1}=\dot{4} \\ 4 k_{2}=\dot{3}\end{array}\right.\right.\right.$

con $k_{1}, k_{2} \in \mathbb{N}$.

Ahora se razona, por ejemplo, sobre $4 k_{2}=\dot{3}$ (también se puede hacer de forma análoga sobre $3 k_{1}=\dot{4}$ ). Se analiza cómo tiene que ser $k_{2}$ para que $4 k_{2}=\dot{3}$. En principio $k_{2}$ puede ser de la forma $\dot{3}, \dot{3}+1$ o $\dot{3}+2$, de manera, que se plantean tres casos:

- Caso I) $k_{2}=3 k_{3} \Rightarrow 4 k_{2}=4 \cdot 3 k_{3}=3 \cdot 4 k_{3}=\dot{3}$.

- Caso II) $k_{2}=3 k_{3}+1 \Rightarrow 4 k_{2}=4\left(3 k_{3}+1\right)=12 k_{3}+4=3\left(4 k_{3}+1\right)+1 \neq \dot{3}$.

- Caso III) $k_{2}=3 k_{3}+2 \Rightarrow 4 k_{2}=4\left(3 k_{3}+2\right)=12 k_{3}+8=3\left(4 k_{3}+2\right)+2 \neq \dot{3}$.

Por lo que si $4 k_{2}=\dot{3}$ solo es posible el caso I para el cual $k_{2}=\dot{3}$. Es decir, se ha probado que $4 k_{2}=\dot{3} \Rightarrow k_{2}=\dot{3}$.

Por tanto $a=4 k_{2}=4 \cdot 3 k_{3}=12 k_{3}=\dot{1}$.

\section{Ejercicios}

1. Sean $a, b, c \in \mathbb{N}$, demostrar que si $a=\dot{b}$ y $b=\dot{c}$ entonces $a=\dot{c}$.

\section{SOLUCIÓN:}

" $\Rightarrow$ " (Directa)

$\left.\begin{array}{l}a=b k_{1} \\ b=c k_{2}\end{array}\right\} \Rightarrow a=b k_{1}=c k_{2} k_{1}=c k_{3} \quad$ con $k_{3} \in \mathbb{N}$

2. Sea $a \in \mathbb{N}$, demostrar que $a=\dot{10} \Leftrightarrow a=\dot{2}$ y $a=\dot{5}$.

\section{SOLUCIÓN:}

" $\Rightarrow$ " (Directa)

$a=10 \Rightarrow a=10 k=2 \cdot 5 k=\left\{\begin{array}{c}2 \cdot(5 k)=2 k^{\prime} \\ 5 \cdot(2 k)=5 k^{\prime \prime}\end{array} \Rightarrow a\right.$ es múltiplo de 2 y $a$ es múltiplo de 5.

$" \Leftarrow "($ Directa $)$ 
$\left\{\begin{array}{l}a=\dot{2} \\ a=\dot{5}\end{array} \Rightarrow\left\{\begin{array}{l}a=2 k_{1} \\ a=5 k_{2}\end{array} \Rightarrow 2 k_{1}=5 k_{2} \Rightarrow\left\{\begin{array}{l}2 k_{1}=\dot{5} \\ 5 k_{2}=\dot{2}\end{array}\right.\right.\right.$

con $k_{1}, k_{2} \in \mathbb{N}$.

Ahora se razona, por ejemplo, sobre $5 k_{2}=\dot{2}$ (también se puede hacer de forma análoga sobre $2 k_{1}=\dot{5}$ ). Se analiza cómo tiene que ser $k_{2}$ para que $5 k_{2}=\dot{2}$. En principio $k_{2}$ puede ser de la forma $\dot{2}$ o $\dot{2}+1$, de manera, que se plantean dos casos:

- Caso I) $k_{2}=2 k_{3} \Rightarrow 5 k_{2}=5 \cdot 2 k_{3}=2 \cdot 5 k_{3}=\dot{2}$.

- Caso II) $k_{2}=2 k_{3}+1 \Rightarrow 5 k_{2}=5\left(2 k_{3}+1\right)=10 k_{3}+5=2\left(5 k_{3}+2\right)+1 \neq \dot{2}$.

Por lo que si $5 k_{2}=\dot{2}$ solo es posible el caso I para el cual $k_{2}=\dot{2}$. Es decir, se ha probado que $5 k_{2}=\dot{2} \Rightarrow k_{2}=\dot{2}$. Por tanto $a=5 k_{2}=5 \cdot 2 k_{3}=10 k_{3}=\dot{10}$.

\subsection{Directas globales}

En las demostraciones directas globales no hay hipótesis explícitas, sino que se asume una hipótesis global ("todo lo que se sabe de un tema").

\section{Ejemplos}

1. Demostrar que la suma de los $n$ primeros números naturales viene dada por la expresión:

$$
1+2+\ldots+n=\frac{n(n+1)}{2} \quad \forall n \in \mathbb{N} .
$$

\section{SOLUCIÓN:}

En realidad lo que se pretende probar es

$$
n \in \mathbb{N} \Rightarrow 1+2+\ldots+n=\frac{n(n+1)}{2} .
$$

“ $\Rightarrow$ (Directa)

Se denota por

$$
S=1+2+\ldots+(n-1)+n
$$

y por tanto, ordenando los sumandos al revés, se tiene que

$$
S=n+(n-1)+\ldots+2+1 .
$$


Sumando ambas expresiones por columnas

$$
\begin{aligned}
2 S & =(1+n)+(2+(n-1))+\ldots+((n-1)+2)+(n+1) \\
& =(n+1)+\ldots(n) \ldots+(n+1)=(n+1) \cdot n .
\end{aligned}
$$

Y por tanto

$$
S=\frac{n(n+1)}{2} .
$$

2. Demostrar que

$$
\forall n, m \in \mathbb{N} \quad\left(\begin{array}{c}
n \\
m
\end{array}\right)+\left(\begin{array}{c}
n \\
m+1
\end{array}\right)=\left(\begin{array}{c}
n+1 \\
m+1
\end{array}\right)
$$

donde $\left(\begin{array}{c}n \\ m\end{array}\right)$ es el número combinatorio $n$ sobre $m$ cuya expresión es

$$
\left(\begin{array}{c}
n \\
m
\end{array}\right)=\frac{n !}{m !(n-m) !}
$$

denotando por $n$ ! al factorial de $n$ que se calcula como $n !=n(n-1)(n-2) \ldots 2 \cdot 1$ y teniendo en cuenta que $0 !=1$.

\section{SOLUCIÓN:}

En realidad hay que probar que

$$
n, m \in \mathbb{N} \Rightarrow\left(\begin{array}{c}
n \\
m
\end{array}\right)+\left(\begin{array}{c}
n \\
m+1
\end{array}\right)=\left(\begin{array}{c}
n+1 \\
m+1
\end{array}\right)
$$

Antes de empezar la demostración se va a probar con algún caso para aprender a manejar estos números combinatorios. Por ejemplo con $n=4$ y $m=2$,

$$
\begin{aligned}
\left(\begin{array}{l}
4 \\
2
\end{array}\right)+\left(\begin{array}{l}
4 \\
3
\end{array}\right) & =\frac{4 !}{2 ! 2 !}+\frac{4 !}{3 ! 1 !}=\frac{4 \cdot 3}{2}+4=6+4=10 \\
\left(\begin{array}{l}
5 \\
3
\end{array}\right) & =\frac{5 !}{3 ! 2 !}=\frac{5 \cdot 4}{2}=10 .
\end{aligned}
$$

Otra forma de razonar es

$$
\left(\begin{array}{l}
4 \\
2
\end{array}\right)+\left(\begin{array}{l}
4 \\
3
\end{array}\right)=\frac{4 !}{2 ! 2 !}+\frac{4 !}{3 ! 1 !}=\frac{4 ! \cdot 3+4 ! \cdot 2}{3 ! 2 !}=\frac{4 !(3+2)}{3 ! 2 !}=\frac{5 !}{3 ! 2 !}=\left(\begin{array}{l}
5 \\
3
\end{array}\right) .
$$

Ahora se procede a la demostración general. 
" $\Rightarrow$ (Directa)

$$
\begin{aligned}
\left(\begin{array}{c}
n \\
m
\end{array}\right)+\left(\begin{array}{c}
n \\
m+1
\end{array}\right) & =\frac{n !}{m !(n-m) !}+\frac{n !}{(m+1) !(n-m-1) !}= \\
& =\frac{n !(m+1)+n !(n-m)}{(m+1) !(n-m) !}=\frac{n !(m+1+n-m)}{(m+1) !(n-m) !}= \\
& =\frac{n !(n+1)}{(m+1) !(n-m) !}=\frac{(n+1) !}{(m+1) !(n-m) !}=\left(\begin{array}{c}
n+1 \\
m+1
\end{array}\right) .
\end{aligned}
$$

Obs.: Dado que el triángulo de Tartaglia está dado por

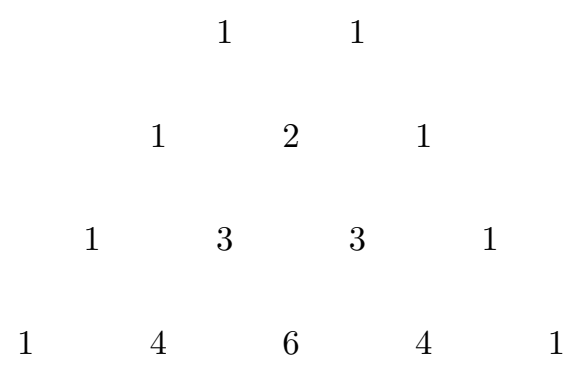

donde cada elemento de una fila es la suma de los dos que la preceden en la fila anterior, este ejercicio ha probado que el elemento $m$-ésimo de la fila $n$-ésima es $\left(\begin{array}{c}n \\ m\end{array}\right)$.

3. Si se define el valor absoluto de un número entero $n$ como

$$
|n|=\left\{\begin{array}{ccc}
n & \text { si } & n \geqslant 0 \\
-n & \text { si } & n<0
\end{array}\right.
$$

demostrar que

$$
\sqrt{n^{2}}=|n| \quad \forall n \in \mathbb{Z}
$$

\section{SOLUCIÓN:}

En realidad lo que se pretende probar es que $n \in \mathbb{Z} \Rightarrow \sqrt{n^{2}}=|n|$. Se distinguen dos casos:

- Caso I) $n \geqslant 0, \sqrt{n^{2}}=\sqrt{n \cdot n}=\sqrt{n} \sqrt{n}=(\sqrt{n})^{2}=n=|n|$.

- Caso II) $n<0, \sqrt{n^{2}}=\sqrt{n \cdot n}=\sqrt{(-n) \cdot(-n)}=\sqrt{-n} \sqrt{-n}=(\sqrt{-n})^{2}=-n=|n|$. Se observa que $\sqrt{-n}$ tiene sentido ya que $n<0$ en el caso II, por lo que $-n>0$. 
Obs.: Otra forma de decir que $\sqrt{n^{2}}=|n|$ es $\sqrt{n^{2}}= \pm n$. No es correcto afirmar que $\sqrt{n^{2}}=n$, ya que por ejemplo $\sqrt{(-4)^{2}}=\sqrt{16}= \pm 4$ y no solo -4 . Evidentemente si $n \in \mathbb{N}$ entonces sí se puede decir que $\sqrt{n^{2}}=n$.

\section{Ejercicios}

1. Demostrar que el valor de la suma de los $n$ primeros números naturales impares es $n^{2}$.

\section{SOLUCIÓN:}

En realidad lo que se pretende probar es

$$
n \in \mathbb{N} \Rightarrow 1+3+5+\ldots+(2 n-3)+(2 n-1)=n^{2} .
$$

“" (Directa)

Se denota por

$$
S=1+3+\ldots+(2 n-3)+(2 n-1)
$$

y por tanto, ordenando los sumandos al revés, se tiene que

$$
S=(2 n-1)+(2 n-3) \ldots+3+1 .
$$

Sumando ambas expresiones por columnas

$$
2 S=(1+2 n-1)+(3+(2 n-3))+\ldots+(2 n-1+1)=\underbrace{2 n+\ldots+2 n}_{n \text { sumandos }}=2 n \cdot n .
$$

Y por tanto $S=n^{2}$.

Obs.: Otra forma de demostrar analíticamente este resultado es utilizar el ejemplo 1:

$$
\begin{aligned}
1+2+3+4+\ldots+(2 n-1)+2 n & =(1+3+\ldots+(2 n-1))+(2+4+\ldots+2 n) \\
\frac{2 n(2 n+1)}{2} & =S+2+4+\ldots+2 n \\
n(2 n+1) & =S+2(1+2+\ldots+n) \\
n(2 n+1) & =S+2 \frac{n(n+1)}{2} \\
2 n^{2}+n & =S+n^{2}+n \\
S & =n^{2} .
\end{aligned}
$$

2. Demostrar que

$$
\forall n \in \mathbb{N} \quad 1+2+\ldots+n=\left(\begin{array}{c}
n+1 \\
n-1
\end{array}\right)
$$




\section{SOLUCIÓN:}

Se tiene que

$$
\begin{aligned}
\left(\begin{array}{l}
n+1 \\
n-1
\end{array}\right) & =\frac{(n+1) !}{(n-1) !(n+1-(n-1)) !}=\frac{(n+1)(n)(n-1) !}{(n-1) ! 2 !} \\
& =\frac{(n+1)(n)}{2 !}=1+2+3+\ldots+n . \quad \square
\end{aligned}
$$

Obs.: Si se denota por $t_{n}=1+2+\ldots+n$, se observa que estos números se pueden disponer geométricamente en forma de triángulo (por eso se llaman números triangulares).

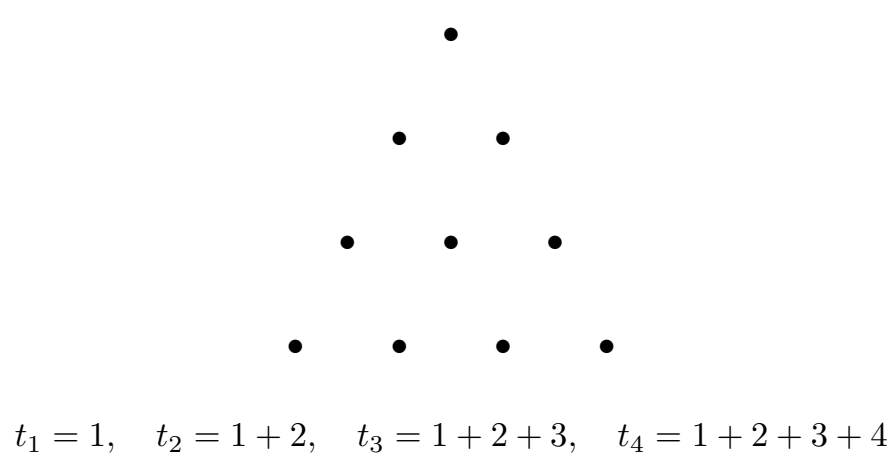

3. Demostrar que

$$
\forall a b \in \mathbb{Z} \quad|a b|=|a||b|
$$

\section{SOLUCIÓN:}

Se consideran cuatro casos:

- Caso I) $a \geqslant 0, b \geqslant 0 ;|a b|=a b=|a||b|$.

- Caso II) $a \geqslant 0, b<0 ; \quad|a b|=-a b=a(-b)=|a||b|$.

- Caso III) $a<0, b \geqslant 0 ;|a b|=-a b=(-a) b=|a||b|$.

- Caso IV) $a<0, b<0 ; \quad|a b|=a b=(-a)(-b)=|a||b|$.

\subsection{Contras}

En las demostraciones a la contra (demostraciones contrarrecíprocas), para demostrar que $A \Rightarrow B$, se demuestra que no $A \Leftarrow$ no $B$, es decir se demuestra que no $B \Rightarrow$ no $A$ (se niega la tesis y se demuestra la negación de la hipótesis). Por otra parte, en las demostraciones a la contra de $A \Leftrightarrow B$ se demuestra que no $A \Leftrightarrow$ no $B$. 


\section{Ejemplos}

1. Sea $k \in \mathbb{N}$, demostrar que si $2^{k}-1$ primo $\Rightarrow k$ primo.

\section{SOLUCIÓN:}

" $\Rightarrow "$ (Directa)

Si se opta por este esquema, no se puede seguir ya que no se tiene una forma genérica de escribir un número primo (a diferencia del ejemplo 1 de la sección "Directas"donde se escribía de forma genérica un número par o en el ejemplo 2 de la sección de "Directas" donde se escribía de forma genérica un múltiplo de 12). Por tanto, hay que intentar otro tipo de demostración.

" $\Rightarrow$ (Contra)

Al utilizarse la demostración a la contra, se va a demostrar que $k$ no primo $\Rightarrow 2^{k}-1$ no primo, o lo que es lo mismo, que $k$ compuesto $\Rightarrow 2^{k}-1$ compuesto. Si se parte de que $k$ es compuesto es porque, de forma genérica, se puede escribir como $k=a b$, por lo que

$$
2^{k}-1=\left(2^{a b}-1\right)=\left(2^{a}\right)^{b}-1=\left(2^{a}-1\right)\left(\left(2^{a}\right)^{b-1}+\left(2^{a}\right)^{b-2}+\ldots+2^{a}+1\right),
$$

donde la última igualdad se demuestra operando y cancelando términos, y así $2^{k}-1$ es producto de dos números $\left(2^{a}-1\right)$ y $\left(\left(2^{a}\right)^{b-1}+\left(2^{a}\right)^{b-2}+\ldots+2^{a}+1\right)$, es decir, $2^{k}-1$ es compuesto.

Obs.: Una vez terminada la demostración es conveniente preguntarse por si será cierto el recíproco, $A \Leftarrow B$, es decir, por si será cierta la afirmación " $2^{k}-1$ primo $\Leftarrow k$ primo", o lo que es lo mismo " $k$ primo $\Rightarrow 2^{k}-1$ primo". Si se empieza a probar con algunos casos se tendría: 2 es primo y $2^{2}-1=3$ es primo, 3 es primo y $2^{3}-1=7$ es primo, 5 es primo y $2^{5}-1=31$ es primo, 7 es primo y $2^{7}-1=127$ es primo, ... Si uno se deja llevar por estos casos se podría intentar demostrar dicha afirmación, utilizando una demostración a la contra (ya que si se parte de que $k$ es primo, de nuevo uno se encuentra con la imposibilidad de dar $k$ de forma genérica). Es decir, se trataría de demostrar que $2^{k}-1$ no primo $\Rightarrow k$ no primo, o sea que $2^{k}-1$ compuesto $\Rightarrow k$ compuesto. Sin embargo, si $2^{k}-1$ compuesto entonces $2^{k}-1=a b$ y aquí el razonamiento se queda bloqueado. Si se hubiese tenido más paciencia, se habría encontrado que $k=11$ cumple que $2^{11}-1=2047$ no es primo ya que $2047=23 \cdot 89$, es decir, se habría encontrado un contraejemplo, con lo que se puede afirmar que $2^{k}-1$ primo $\nLeftarrow k$ primo.

2. Sea $a \in \mathbb{N}$, demostrar que $a^{2}$ impar $\Leftrightarrow a$ impar.

\section{SOLUCIÓN:}

" $\Rightarrow$ (Directa)

Dado que $a^{2}$ es impar, se puede escribir $a^{2}=2 k+1, k \in \mathbb{N}$, por lo que $a=\sqrt{2 k+1}$ y aquí el razonamiento se 
queda bloqueado. Hay, por tanto, que intentar otro tipo de demostración.

" $\Rightarrow "($ Contra $)$

Se trata de probar que $a^{2}$ no impar $\Leftarrow a$ no impar. Es decir que $a$ par $\Rightarrow a^{2}$ par. Y esto es cierto utilizando el ejemplo 1 de la sección de "Directaçon $b=a$.

$" \Leftarrow "($ Directa $)$

Se parte de $a$ impar, $a=2 k+1, k \in \mathbb{N}$, con lo que $a^{2}=(2 k+1)^{2}=4 k^{2}+4 k+1=2\left(2 k^{2}+2 k\right)+1$ es impar.

Obs: En este caso la demostración " $\Leftarrow$ " (Contra) se quedaría bloqueada.

\section{Ejercicios}

1. Demostrar que $\forall k \in \mathbb{N}$

$$
4 k=\dot{3} \Rightarrow k=\dot{3} .
$$

\section{SOLUCIÓN:}

Se trata de probar que

$$
k \neq \dot{3} \Rightarrow 4 k \neq \dot{3}
$$

Si $k \neq \dot{3}$ entonces $\exists k_{1} \in \mathbb{N}$ tal que $k=3 k_{1}+1$ o $k=3 k_{1}+2$. En el primer caso $4 k=4\left(3 k_{1}+1\right) \neq \dot{3}$ y en el segundo $4 k=4\left(3 k_{1}+2\right) \neq \dot{3}$.

2. Sea $a \in \mathbb{N}$, demostrar que

$$
a^{2} \operatorname{par} \Leftrightarrow a \text { par. }
$$

\section{SOLUCIÓN:}

" $\Rightarrow$ (Directa)

Dado que $a^{2}$ es par, se puede escribir $a^{2}=2 k, \quad k \in \mathbb{N}$, por lo que $a=\sqrt{2 k}$ y aquí el razonamiento se queda bloqueado.

" $\Rightarrow$ " (Contra)

Se trata de probar que $a^{2}$ no par $\Leftarrow a$ no par. Es decir que $a$ impar $\Rightarrow a^{2}$ impar, que ha sido demostrado en el ejemplo 2 de esta sección.

"६" (Directa)

Esto ya está demostrado en el ejemplo 1 de la sección 1.1 con $b=a$.

Obs.: El resultado $a^{2}$ par $\Leftrightarrow a$ par se podría haber demostrado directamente probando $a^{2}$ no par $\Leftrightarrow a$ no par, que es el ejemplo 2. 


\section{Capítulo 2}

\section{Necesaria y suficiente}

\section{Objetivos}

- Conocer ejemplos concretos de condiciones necesarias y suficientes.

- Saber expresar en términos del lenguaje habitual dichas condiciones.

En este mini-capítulo se describen los conceptos de condición necesaria y suficiente asociadas a afirmaciones del tipo $A \Rightarrow B$. En la sección 2.1 se explica lo relativo al concepto de condición necesaria y en la sección 2.2 lo relativo a condición suficiente.

Es conveniente ver el MDM "Necesaria y suficiente" antes de seguir leyendo este mini-capítulo.

\subsection{Necesaria}

En el esquema $A \Rightarrow B$, se dice que $B$ es condición necesaria para $A$, y se expresa diciendo que si se da $A$ necesariamente se da $B$. Por ejemplo "llueve $\Rightarrow$ calle mojada" por lo que "calle mojada. es condición necesaria para "llueve", es decir, que si "llueve", necesariamente "calle mojada".

\section{Ejemplos}

1. Demostrar que para un número primo $p, p>3$, necesariamente se da que $p^{2}-1=1 \dot{2}$, es decir, que $p^{2}-1=1 \dot{2}$ con $p>3$ es condición necesaria para que $p$ sea primo (ver [1])

\section{SOLUCIÓN:}

El enunciado de este ejemplo se representa por

$$
p>3, \text { p primo } \Rightarrow p^{2}-1=\dot{1} \text {. }
$$


" $\Rightarrow "($ Directa $)$

Aunque no se tenga una forma genérica de escribir un número primo $p>3$, se puede razonar a partir de que cualquier número es de la forma $\dot{3}, \dot{3}+1$ o $\dot{3}+2$. Como en el enunciado se parte de que $p$ es primo, la situación $\dot{3}$ no se puede dar (porque entonces $p$ sería compuesto) y por tanto se tiene que $p$ es $\dot{3}+1$ o $\dot{3}+2$, donde además en ambos casos $p$ tiene que ser impar (dado que $p$ es primo, con $p>3$ ). En cualquiera de las situaciones: $p$ impar $\Rightarrow p+1$ es par y $p-1$ es par $\Rightarrow(p+1)(p-1)=\dot{4} \Rightarrow p^{2}-1=\dot{4}$.

Ahora se trata de razonar por casos para demostrar el resultado pedido:

- Caso I) $p=\dot{3}+1, p$ impar. Se tiene $p=\dot{3}+1 \Rightarrow p-1=\dot{3}+1-1=\dot{3} \Rightarrow(p+1)(p-1)=\dot{3} \Rightarrow p^{2}-1=\dot{3}$.

- Caso II) $p=\dot{3}+2, p$ impar. Se tiene $p=\dot{3}+2 \Rightarrow p+1=\dot{3}+2+1=\dot{3}+3=\dot{3} \Rightarrow(p+1)(p-1)=\dot{3} \Rightarrow$ $p^{2}-1=\dot{3}$.

En ambos casos, por el ejemplo 2 de la sección 1.1 , se verifica que $p^{2}-1=\dot{4}$ y $p^{2}-1=\dot{3} \Rightarrow p^{2}-1=1 \dot{2}$.

Obs. 1 Terminada la demostración es conveniente preguntarse por si será cierto el recíproco $A \Leftarrow B$, es decir, por si $B \Rightarrow A(A$ es necesaria para $B)$ o sea que si se da $B$ entonces necesariamente se da $A$. En definitiva, que si se cumple que $p^{2}-1=1 \dot{2}$, entonces $p$ primo. Como antes, se prueba con algunos casos. Del conjunto de múltiplos de $12:\{12,24,36,48,60, \ldots\}$ se extraen aquellos que son de la forma $p^{2}-1:\left\{24=5^{2}-1,48=7^{2}-1, \ldots\right\}$ y parece que la afirmación es factible. Sin embargo algo más de tenacidad permite encontrar $4190208=1 \dot{2}$ y que verifica $2047^{2}-1=4190208$ con 2047 no primo, ya que $2047=23 \cdot 89$. Es decir $p^{2}-1=1 \dot{2} \nRightarrow p$ primo.

Obs. 2 A la vista de la Obs. 1 se puede afirmar que "si $p^{2}-1=\dot{1} \dot{2}$, no se tiene que necesariamente $p$ sea primo". Sin embargo, si $p^{2}-1 \neq 1 \dot{2}$ ya se puede afirmar que $p$ no es primo, con lo que se tiene un procedimiento para saber si un número es compuesto sin haber comprobado si tiene o no divisores. El procedimiento solo funciona si $p^{2}-1 \neq 1 \dot{2}$ ya que si $p^{2}-1=1 \dot{2}$ no se puede afirmar nada.

2. Demostrar que si el cuadrado de un número natural es múltiplo de 3 , necesariamente se da que dicho número es múltiplo de 3 . Es decir, que $a=\dot{3}$ es condición necesaria para que $a^{2}=\dot{3}$.

\section{SOLUCIÓN:}

El enunciado de este ejemplo se representa por

$$
\forall a \in \mathbb{N} \quad a^{2}=\dot{3} \Rightarrow a=\dot{3}
$$

" $\Rightarrow "($ Directa $)$

Si se supone que $a^{2}=3 k$ con $k \in \mathbb{N}$, se observa que el razonamiento queda bloqueado ya que no se puede concluir nada a partir de $\sqrt{a}$. 
" $\Rightarrow "($ Contra $)$

¿ $a^{2}$ no $\dot{3} \Leftarrow a$ no $\dot{3}$ ?, o bien, ¿a no $\dot{3} \Rightarrow a^{2}$ no $\dot{3}$ ?

Se razona por casos:

- Caso I) $a=3 k+1 \Rightarrow a^{2}=(3 k+1)(3 k+1)=9 k^{2}+3 k+3 k+1=3\left(3 k^{2}+2 k\right)+1 \neq \dot{3}$.

- Caso II) $a=3 k+2 \Rightarrow a^{2}=(3 k+2)(3 k+2)=9 k^{2}+6 k+6 k+4=3\left(3 k^{2}+4 k+1\right)+1 \neq \dot{3}$.

Obs. Terminada la demostración es conveniente preguntarse por si será cierto el recíproco $A \Leftarrow B$, es decir, por si $B \Rightarrow A(A$ es necesaria para $B)$, por si se da $B$ entonces necesariamente se da $A$. En definitiva si se cumple que $a=\dot{3} \Rightarrow a^{2}=\dot{3}$.

$" \Rightarrow "($ Directa $)$

En este caso la demostración directa no se queda bloqueada como antes: $a=3 k, k \in \mathbb{N} \Rightarrow a^{2}=9 k^{2}=3\left(3 k^{2}\right)=$ $3 k^{\prime}=\dot{3}$.

En este ejemplo se da que

$$
\begin{aligned}
& a=\dot{3} \text { es necesaria para } a^{2}=\dot{3} . \\
& a^{2}=\dot{3} \text { es necesaria para } a=\dot{3} .
\end{aligned}
$$

3. Demostrar que si $n \in \mathbb{Z}, a \in \mathbb{N}$ con $|n|<a$, necesariamente se da que $-a<n<a$. Es decir que $n \in \mathbb{Z}, a \in \mathbb{N}$ con $-a<n<a$ es condición necesaria para que $|n|<a$.

\section{SOLUCIÓN:}

El enunciado de este ejemplo se representa por

$$
n \in \mathbb{Z} a \in \mathbb{N}, \quad|n|<a \Rightarrow-a<n<a .
$$

$" \Rightarrow "($ Directa $)$

$$
\left.\begin{array}{rl}
n & \leqslant \\
-n & \leqslant
\end{array}\right\} \stackrel{n \mid}{\stackrel{|n|<a}{\Rightarrow}}\left\{\begin{array} { c } 
{ n < a } \\
{ - n < a }
\end{array} \Rightarrow \left\{\begin{array}{c}
n<a \\
n>-a
\end{array} \Rightarrow-a<n<a .\right.\right.
$$

Obs. Es fácil ver que en este caso, también se da el recíproco 
" " (Directa)

$$
-a<n<a \Rightarrow\left\{\begin{array} { c } 
{ n < a } \\
{ - a < n }
\end{array} \Rightarrow \left\{\begin{array} { c } 
{ n < a } \\
{ a > - n }
\end{array} \Rightarrow \left\{\begin{array}{c}
n<a \\
-n<a
\end{array} \Rightarrow|n|<a .\right.\right.\right.
$$

\section{Ejercicios}

1. Demostrar que si $a \in \mathbb{Z}, b, c \in \mathbb{N}, a^{2} \leqslant b c$, necesariamente se da que $|a| \leqslant \sqrt{b} \sqrt{c}$.

\section{SOLUCIÓN:}

El enunciado de este ejercicio se representa por

$$
a \in \mathbb{Z}, b, c \in \mathbb{N}, a^{2} \leqslant b c \Rightarrow|a| \leqslant \sqrt{b} \sqrt{c} .
$$

" $\Rightarrow "($ Directa $)$

$$
a^{2} \leqslant b c \Rightarrow \sqrt{a^{2}} \leqslant \sqrt{b c} \Rightarrow|a| \leqslant \sqrt{b} \sqrt{c}
$$

Obs. Es fácil ver que en este caso, también se da el recíproco

$" \Leftarrow "($ Directa $)$

$$
|a| \leqslant \sqrt{b} \sqrt{c} \Rightarrow|a|^{2} \leqslant(\sqrt{b} \sqrt{c})^{2} \Rightarrow a^{2} \leqslant b c
$$

2. Demostrar que ser múltiplo de 2 y de 3 es condición necesaria para ser múltiplo de 6 .

\section{SOLUCIÓN:}

El enunciado de este ejercicio se representa por

$$
a=\dot{6} \Rightarrow a=\dot{2} \text { y } \quad a=\dot{3}
$$

$" \Rightarrow "($ Directa $)$

$$
a=\dot{6} \Rightarrow a=6 k=\left\{\begin{array}{r}
3(2 k)=\dot{3} \\
2(3 k)=\dot{2}
\end{array}\right.
$$

Obs. Es fácil ver que en este caso también se da el recíproco, según se mostrará en la sección 2.2 .

3. Demostrar que si $a, b \in \mathbb{Z}, a^{2} \leqslant b^{2}$, necesariamente se da que $|a| \leqslant|b|$.

\section{SOLUCIÓN:}


El enunciado de este ejercicio se representa por

$$
a, b \in \mathbb{Z}, a^{2} \leqslant b^{2} \Rightarrow|a| \leqslant|b| .
$$

$" \Rightarrow "($ Directa $)$

$$
a^{2} \leqslant b^{2} \Rightarrow \sqrt{a^{2}} \leqslant \sqrt{b^{2}} \Rightarrow|a| \leqslant|b| .
$$

Obs. Es fácil ver que en este caso, también se da el recíproco

$" \Leftarrow "($ Directa $)$

$$
|a| \leqslant|b| \Rightarrow|a|^{2} \leqslant|b|^{2} \Rightarrow a^{2} \leq b^{2}
$$

\subsection{Suficiente}

En el esquema $A \Rightarrow B$, se dice que $A$ es condición suficiente para $B$, y se expresa como "para que se cumpla $B$ es suficiente que se cumpla $A$ ". Por ejemplo "llueve $\Rightarrow$ calle mojada", por lo que para que haya calle mojada es suficiente con que llueva.

Puede ocurrir que además de $A \Rightarrow B$ se dé que $B \Rightarrow A$, en cuyo caso se dice que $A$ es condición necesaria y suficiente para $B$ y, al revés, que $B$ es condición necesaria y suficiente para $A$. También puede suceder que $A \Rightarrow B$ pero $B \nRightarrow A$, como en el caso llueve $\Rightarrow$ calle mojada pero calle mojada $\nRightarrow$ llueve (por ejemplo en el caso de que hayan regado se tiene la calle mojada), con lo que $B$ es necesaria para $A$ pero $B$ no es suficiente para $A$ (por ejemplo, calle mojada no es condición suficiente para que llueva, o que llueva no es necesaria para calle mojada).

\section{Ejemplos}

1. Demostrar que si $p>3$ verifica que $p^{2}-1 \neq 1 \dot{2}$, eso es suficiente para afirmar que $p$ no es primo.

\section{SOLUCIÓN:}

El enunciado de este ejemplo se representa por

$$
p>3, p^{2}-1 \neq 1 \dot{2} \Rightarrow p \text { no primo }
$$

que corresponde a la observación 2 del ejemplo 1 de la sección anterior.

Obs: En Matemáticas conviene tener condiciones necesarias y suficientes, aunque a veces, desgraciadamente, no sean de utilidad práctica. Esto ocurre con el Teorema de Wilson que afirma que para que $p$ sea primo es condición necesaria y suficiente que $(p-1) !+1=\stackrel{\bullet}{p}$. Con dicho teorema, incluso para ver que un número pequeño es primo, al evaluar el factorial aparecen números muy grandes: por ejemplo, para comprobar que 17 es primo hay que operar $16 !+1=20922789888001$ y verificar que es múltiplo de 17 . 
2. Demostrar que si un número natural es múltiplo de 3, eso es suficiente para decir que su cuadrado también lo es.

\section{SOLUCIÓN:}

El enunciado de este ejemplo se representa por

$$
\forall a \in \mathbb{N}, a=\dot{3} \Rightarrow a^{2}=\dot{3}
$$

$" \Rightarrow "($ Directa $)$

$$
a=3 k \Rightarrow a^{2}=9 k^{2}=3\left(3 k^{2}\right)=\dot{3}
$$

Obs: Como $a=\dot{3}$ también es condición necesaria para que $a^{2}=\dot{3}$, se tiene que $a=\dot{3}$ es condición necesaria y suficiente para que $a^{2}=\dot{3}$, o sea $a^{2}=\dot{3} \Leftrightarrow a=\dot{3}$.

\section{Ejercicios}

1. Demostrar que es suficiente que $a \in \mathbb{Q}, 0<a<1$ para que $a^{2}<1$.

\section{SOLUCIÓN:}

El enunciado de este ejercicio se representa por

$$
a \in \mathbb{Q}, 0<a<1 \Rightarrow a^{2}<1
$$

$" \Rightarrow "($ Directa $)$

$$
0<a<1 \Rightarrow a<1 \stackrel{a>0}{\Rightarrow} a \cdot a<a \cdot 1 \Rightarrow a^{2}<a<1
$$

Obs: Sin embargo no es condición necesaria porque $a \in \mathbb{Q}, a^{2}<1 \nRightarrow 0<a<1$. como se puede ver, por ejemplo, con $a=-1 / 2$.

2. Demostrar que $a=\dot{2}$ y $a=\dot{3}$ es condición suficiente para que $a=\dot{6}$.

\section{SOLUCIÓN:}

El enunciado de este ejercicio se representa por

$$
a=\dot{2} \text { у } a=\dot{3} \Rightarrow a=\dot{6}
$$

" $\Rightarrow "($ Directa $)$

$$
\left.\begin{array}{l}
a=2 k_{1} \text { con } k_{1} \in \mathbb{N} \\
a=3 k_{2} \operatorname{con} k_{2} \in \mathbb{N}
\end{array}\right\} \Rightarrow 2 k_{1}=3 k_{2} \Rightarrow k_{2}=\frac{2 k_{1}}{3} \Rightarrow k_{1}=\dot{3}
$$


Esta última implicación se demuestra por contra distinguiendo dos casos:

- Caso I) $k_{1}=3 k+1 \Rightarrow \frac{2(3 k+1)}{3}=2 k+\frac{2}{3} \notin \mathbb{N}$, lo que supone una contradicción.

- Caso II) $k_{1}=3 k+2 \Rightarrow \frac{2(3 k+2)}{3}=2 k+\frac{4}{3} \notin \mathbb{N}$, y de nuevo se produce una contradicción.

Retomando la argumentación $a=2 k_{1}=2 \cdot 3 k=\stackrel{\bullet}{6}$ con $k \in \mathbb{N}$.

Obs. Como además $a=\dot{2}$ y $a=\dot{3}$ es condición necesaria para que $a=\dot{6}$, se tiene que $a=\dot{2}$ y $a=\dot{3}$ es condición necesaria y suficiente para que $a=\dot{6}$, es decir $a=\dot{6} \Leftrightarrow a=\dot{2}$ y $a=\dot{3}$. 


\section{Capítulo 3}

\section{Supongamos que}

\section{Objetivos}

- Exponer ejemplos concretos de demostraciones por reducción al absurdo y por marcha hacia atrás.

- Conocer la existencia de números no racionales.

En este mini-capítulo se describe la reducción al absurdo (que no conviene confundir con demostración a la contra) y marcha hacia atrás.

Es conveniente ver los MDM " Reducción al absurdo"] y "Marcha hacia atrás"] antes de seguir leyendo este minicapítulo.

\subsection{Reducción al absurdo}

La idea para demostrar la implicación $A \Rightarrow B$ por reducción al absurdo, consiste en suponer simultáneamente ciertas las proposiciones $A$ y no $B$, y a partir de esta suposición, usando deducciones matemáticas, llegar a una contradicción o algo absurdo, con lo que se habría comprobado que la proposición $A \Rightarrow B$ es cierta. En otras ocasiones la reducción al absurdo se aplica a demostraciones directas globales donde no hay hipótesis explícitas, sino que se asume una hipótesis global.

No confundir con el razonamiento de demostración a la contra (no $B \Rightarrow$ no $A$ ).

\section{Ejemplos}

1. Demostrar que $\sqrt{2}$ no es un número racional.

\section{SOLUCIÓN:}

Se supone que $\sqrt{2}$ es un número racional, de manera que se puede expresar como $\sqrt{2}=\frac{a}{b}$, con $a, b \in \mathbb{N}$ siendo $\frac{a}{b}$ 
una fracción irreducible, es decir, que $a$ y $b$ no tienen ningún factor común, ya que en el caso de que lo tuvieran se podría simplificar y así hasta que fuera irreducible. Operando $a^{2}=2 b^{2} \Rightarrow a^{2}=\dot{2}$ y por el ejercicio 2 de la sección 1.3. se tiene que $a=\dot{2} \Rightarrow a=2 c$ con $c \in \mathbb{N}$ y sustituyendo en la igualdad anterior, resulta $4 c^{2}=2 b^{2}$, es decir, $2 c^{2}=b^{2}$, con lo que también $b$ es par. Esto lleva a una contradicción puesto que se había partido de que $a$ y $b$ no tenían ningún factor común. Por tanto el punto de partida, es decir que $\sqrt{2}$ es racional es falso.

2. Demostrar que si $a^{2}+b^{2}=\dot{3}$ con $a, b \in \mathbb{N}$ entonces $a=\dot{3}$ y $b=\dot{3}$.

\section{SOLUCIÓN:}

La demostración se hace por reducción al absurdo. Se supone que $a^{2}+b^{2}=\dot{3}$ es cierto y que $a=\dot{3}$ y $b=\dot{3}$ es falso, es decir, $a \neq \dot{3} \quad$ o $\quad b \neq \dot{3}$.

Si se considera que es $a \neq \dot{3}$ (si fuera $b$ sería análogo), las posibilidades son

$$
\begin{aligned}
& a=3 k+1 \text { y } \quad\left(b=3 k^{\prime} \text { о } b=3 k^{\prime}+1 \text { о } b=3 k^{\prime}+2\right) \\
& a=3 k+2 \text { у } \quad\left(b=3 k^{\prime} \text { о } b=3 k^{\prime}+1 \text { о } b=3 k^{\prime}+2\right) .
\end{aligned}
$$

que se pueden escribir como

$$
\begin{aligned}
& a=3 k+i, \quad i=1,2 \\
& b=3 k^{\prime}+j, \quad j=0,1,2 .
\end{aligned}
$$

Entonces

$$
\begin{aligned}
a^{2}+b^{2} & =(3 k+i)^{2}+\left(3 k^{\prime}+j\right)^{2}=9 k^{2}+i^{2}+6 k i+9 k^{2}+j^{2}+6 k^{\prime} j \\
& =3 \underbrace{\left(3 k^{2}+2 k i+3 k^{\prime 2}+2 k^{\prime} j\right)}_{k^{\prime \prime}}+\underbrace{i^{2}+j^{2}}_{l}=3 k^{\prime \prime}+l
\end{aligned}
$$

donde

$$
\begin{aligned}
l & =i^{2}+j^{2} \in\left\{1^{2}+0^{2}=1,1^{2}+1^{2}=2,1^{2}+2^{2}=5,2^{2}+0^{2}=4,2^{2}+1^{2}=5,2^{2}+2^{2}=8\right\} \\
& =\{1,2,5,4,5,8\} \neq \dot{3} .
\end{aligned}
$$

con lo que $l=3 l^{\prime}+1$ o $l=3 l^{\prime}+2$. Por tanto $a^{2}+b^{2}=3 k^{\prime \prime}+3 l^{\prime}+1$ o $a^{2}+b^{2}=3 k^{\prime \prime}+3 l^{\prime}+2$.

Luego $a^{2}+b^{2} \neq \dot{3}$, lo que produce una contradicción con el enunciado. 


\section{Ejercicios}

1. Demostrar que $\sqrt{6}$ no es un número racional.

\section{SOLUCIÓN:}

Se supone que $\sqrt{6}$ es un número racional, de manera que se puede expresar como $\sqrt{6}=\frac{a}{b}$, con $a, b \in \mathbb{N}$ siendo $\frac{a}{b}$ una fracción irreducible, es decir, que $a$ y $b$ no tienen ningún factor común. Entonces $a^{2}=6 b^{2}$, lo que lleva a que $a^{2}=\dot{6}$, y esto equivale a que $a^{2}=\dot{2}$ y $a^{2}=\dot{3}$. Pero por el ejercicio 2 de la sección 2.1. lo anterior implica $a=\dot{2}$ y $a=\dot{3}$, luego $a=\dot{6}$ y se puede expresar $a=6 k$. Sustituyendo en la igualdad $a^{2}=6 b^{2}$, resulta $6^{2} k^{2}=6 b^{2} \Rightarrow b^{2}=6 k^{2} \Rightarrow b=\dot{6}$. Esto conduce a una contradicción puesto que se había partido de que $a$ y $b$ no tenían ningún factor común y ahora se ha visto que ambos eran múltiplos de 6 . Por tanto el punto de partida, es decir que $\sqrt{6}$ es racional es falso.

2. Demostrar que si $a^{2}+b^{2}+a b=\stackrel{\bullet}{10}$ con $a, b \in \mathbb{N}$ entonces $a=\stackrel{\bullet}{10}$ y $b=\stackrel{\bullet}{10}$ (ver [3] y [4]).

\section{SOLUCIÓN:}

En primer lugar se observa que $a$ y $b$ son pares, ya que si uno de ellos fuera impar es fácil ver que $a^{2}+b^{2}+a b$ sería impar y no podría ser múltiplo de 10 . Como $a$ y $b$ son pares, para probar que $a=10$ y $b=10$, hay que verificar que $a=\dot{5}$ y $b=\dot{5}$, lo que se hará por reducción al absurdo. Se supone que $a \neq \dot{5}$ o $b \neq \dot{5}$, es decir, $a=5 k+i$ y $b=5 k^{\prime}+j$, donde $i \neq 0$ o $j \neq 0$. A continuación se observa que $a^{2}+b^{2}+a b$ $=25\left(k^{2}+k^{\prime 2}+k k^{\prime}\right)+5\left(2 k i+2 k^{\prime} j+k j+k^{\prime} i\right)+\left(i^{2}+j^{2}+i j\right)=5 k^{\prime \prime}+l$ donde los posibles valores de $l$ se calculan en la tabla 3.1 obviándose los casos simétricos en $i$ y en $j$. Por lo tanto, $l \neq \dot{5}$, con lo que $a^{2}+b^{2}+a b \neq \dot{5}$ lo que entraría en contradicción con que $a^{2}+b^{2}+a b=\dot{10}$. Luego la hipótesis de partida $a \neq \dot{5}$ o $b \neq \dot{5}$ es falsa.

\subsection{Marcha hacia atrás}

En el método de marcha hacia atrás se actúa como sigue: el resultado a demostrar se transforma, en sucesivos pasos transitorios, en expresiones cada vez más simples hasta llegar a una lo suficientemente sencilla para que su demostración sea inmediata. Al finalizar el proceso es conveniente realizar la demostración de forma directa.

\section{Ejemplos}

1. Demostrar que si $x, y \in \mathbb{R}$ con $x, y>0$, entonces

$$
\frac{2}{\frac{1}{x}+\frac{1}{y}} \leqslant \sqrt{x y} .
$$

\section{SOLUCIÓN:}

La demostración se hace por "marcha hacia atrás", por lo que se opera la expresión 


\begin{tabular}{|c|c|c|}
\hline$i$ & $j$ & $l$ \\
\hline 0 & 1 & 1 \\
0 & 2 & 4 \\
0 & 3 & 9 \\
0 & 4 & 16 \\
\hline 1 & 1 & 3 \\
1 & 2 & 7 \\
1 & 3 & 13 \\
1 & 4 & 21 \\
\hline 2 & 2 & 12 \\
2 & 3 & 19 \\
2 & 4 & 28 \\
\hline 3 & 3 & 27 \\
3 & 4 & 37 \\
\hline 4 & 4 & 48 \\
\hline
\end{tabular}

Cuadro 3.1: Posibles valores de $l=i^{2}+j^{2}+i j$

$$
\frac{2}{\frac{1}{x}+\frac{1}{y}} \leqslant \sqrt{x y}
$$

obteniéndose que

$$
\begin{aligned}
& \frac{2 x y}{y+x} \leqslant \sqrt{x y} \\
& \left(\frac{2 x y}{y+x}\right)^{2} \leqslant(\sqrt{x y})^{2} \\
& \frac{4 x^{2} y^{2}}{(y+x)^{2}} \leqslant|x y| \\
& 4 x^{2} y^{2} \leqslant(y+x)^{2}|x y| .
\end{aligned}
$$

(Obsérvese que la última desigualdad no cambia de sentido ya que $(y+x)^{2} \geqslant 0$ ).

Con lo que si se logra probar que

$$
x, y>0 \Rightarrow 4 x^{2} y^{2} \leqslant(y+x)^{2}|x y|
$$


se tendría el resultado. En el supuesto de $x, y>0$, lo anterior es equivalente a probar

$$
\begin{aligned}
4 x^{2} y^{2} & \leqslant\left(x^{2}+y^{2}+2 x y\right) x y \\
4 x^{2} y^{2} & \leqslant x^{3} y+x y^{3}+2 x^{2} y^{2} \\
0 & \leqslant x^{3} y+x y^{3}-2 x^{2} y^{2} \\
0 & \leqslant x y\left(x^{2}+y^{2}-2 x y\right) \\
0 & \leqslant x y(x-y)^{2} .
\end{aligned}
$$

Y esto último se prueba que es cierto directamente, ya que por una parte $(x-y)^{2} \geqslant 0$ siempre y por otra $x y>0$, ya que se está suponiendo que $x, y>0$.

En estos momentos quizás sea interesante para reforzar ideas reescribir la demostración de forma directa reconstruyéndola (se gana claridad aunque se pierde la intuitividad que da el método de "marcha hacia atrás"):

$$
\begin{aligned}
& x, y>0 \Rightarrow x y(x-y)^{2} \geqslant 0 \Rightarrow x y\left(x^{2}+y^{2}-2 x y\right) \geqslant 0 \Rightarrow \\
& x^{3} y+x y^{3}-2 x^{2} y^{2} \geqslant 0 \Rightarrow x^{3} y+x y^{3}+2 x^{2} y^{2} \geqslant 4 x^{2} y^{2} \Rightarrow \\
& \left(x^{2}+y^{2}+2 x y\right) x y \geqslant 4 x^{2} y^{2} \Rightarrow x y \geqslant \frac{4 x^{2} y^{2}}{(y+x)^{2}} \Rightarrow \sqrt{x y} \geqslant \frac{\sqrt{4 x^{2} y^{2}}}{\sqrt{(y+x)^{2}}} \Rightarrow \\
& \sqrt{x y} \geqslant \frac{2|x y|}{|y+x|} \stackrel{x, y>0}{\Rightarrow} \sqrt{x y} \geqslant \frac{2 x y}{y+x} \Rightarrow \sqrt{x y} \geqslant \frac{2}{\frac{1}{x}+\frac{1}{y}} .
\end{aligned}
$$

2. Demostrar que si $a, b, c \in \mathbb{R}$ con $a, b, c>0$, entonces

$$
3(a b+b c+c a) \leqslant(a+b+c)^{2} .
$$

\section{SOLUCIÓN:}

La demostración se hace por "marcha hacia atrás", por lo que se opera la expresión

$$
3(a b+b c+c a) \leqslant(a+b+c)^{2}
$$

obteniéndose que

$$
\begin{aligned}
3 a b+3 b c+3 c a & \leqslant a^{2}+b^{2}+c^{2}+2 a b+2 a c+2 b c \\
a b+b c+c a & \leqslant a^{2}+b^{2}+c^{2} .
\end{aligned}
$$


Con lo que si se logra probar que $a, b, c>0 \Rightarrow a b+b c+c a \leqslant a^{2}+b^{2}+c^{2}$ se tendrá el resultado.

Se supone por tanto, $a, b, c>0$ y sin perdida de generalidad que $a \geqslant b \geqslant c>0$ (los otros casos serán análogos), y se pretende probar que $a b+b c+c a \leqslant a^{2}+b^{2}+c^{2}$. Realizándose el siguiente cambio de notación $x=\frac{b}{a}, y=\frac{c}{a}$ (es posible dividir por $a$, ya que $a>0$ ), se está suponiendo que $1 \geqslant x \geqslant y>0$ y se intenta demostrar que $a b+b c+c a \leqslant a^{2}+b^{2}+c^{2}$ que equivale a

$$
\begin{aligned}
\frac{a b+b c+c a}{a^{2}} & \leqslant \frac{a^{2}+b^{2}+c^{2}}{a^{2}} \\
\frac{b}{a}+\frac{c}{a}+\frac{b c}{a^{2}} & \leqslant 1+\frac{b^{2}}{a^{2}}+\frac{c^{2}}{a^{2}} \\
x+y+x y & \leqslant 1+x^{2}+y^{2} \\
0 & \leqslant x^{2}+y^{2}+1-x-y-x y \\
0 & \leqslant\left(x^{2}+y^{2}-2 x y\right)+(1-x-y+x y) \\
0 & \leqslant(x-y)^{2}+(1-x)(1-y) .
\end{aligned}
$$

Y esto último se prueba que es cierto directamente, ya que por una parte $(x-y)^{2} \geqslant 0$ siempre y además por otra parte $(1-x)(1-y) \geqslant 0$, ya que se está suponiendo que $1-x \geqslant 0,1-y \geqslant 0$.

\section{Ejercicios}

1. Demostrar que si $x \in \mathbb{R}$ con $x>0$, entonces

$$
x+\frac{1}{x} \geqslant 2
$$

\section{SOLUCIÓN:}

La demostración se hace por "marcha hacia atrás", por lo que se opera la expresión $x+\frac{1}{x} \geqslant 2$ obteniéndose que

$$
\frac{x^{2}+x}{x} \geqslant 2
$$

Con lo que si se logra probar que $x>0 \Rightarrow \frac{x^{2}+x}{x} \geqslant 2$ se tendrá el resultado. Se supone, por tanto, que $x>0 \mathrm{y}$ se intenta demostrar que $\frac{x^{2}+x}{x} \geqslant 2$, que en el supuesto de $x>0$ es equivalente a probar

$$
\begin{aligned}
x^{2}+x & \geqslant 2 x \\
x^{2}+x-2 x & \geqslant 0 \\
(x-1)^{2} & \geqslant 0 .
\end{aligned}
$$

Y esto último se prueba que es cierto directamente, ya que el cuadrado de un número es siempre mayor o igual 
que cero.

En este ejercicio es muy sencillo reconstruir la demostración de forma directa:

$$
(x-1)^{2} \geqslant 0 \Rightarrow x^{2}+x-2 x \geqslant 0 \Rightarrow x^{2}+x \geqslant 2 x \stackrel{x>0}{\Rightarrow} \frac{x^{2}+x}{x} \geqslant 2 \Rightarrow x+\frac{1}{x} \geqslant 2 .
$$

2. Demostrar que si $x, y \in \mathbb{R}$ con $x, y>0$, entonces

$$
\sqrt{x y} \leqslant \frac{x+y}{2}
$$

\section{SOLUCIÓN:}

La demostración se hace por "marcha hacia atrás", por lo que se opera la expresión

$$
\sqrt{x y} \leqslant \frac{x+y}{2}
$$

obteniendo que

$$
\begin{aligned}
(\sqrt{x y})^{2} & \leqslant\left(\frac{x+y}{2}\right)^{2} \\
|x y| & \leqslant \frac{(x+y)^{2}}{4}
\end{aligned}
$$

Con lo que si se logra probar que $x, y>0 \Rightarrow|x y| \leqslant \frac{(x+y)^{2}}{4}$ se tendrá el resultado.

Se supone, por tanto, que $x, y>0$ y se intenta demostrar que $|x y| \leqslant \frac{(x+y)^{2}}{4}$, que en el supuesto de $x, y>0$ es equivalente a probar

$$
\begin{aligned}
x y & \leqslant \frac{x^{2}+y^{2}+2 x y}{4} \\
4 x y & \leqslant x^{2}+y^{2}+2 x y \\
0 & \leqslant x^{2}+y^{2}-2 x y \\
0 & \leqslant(x-y)^{2} .
\end{aligned}
$$

$\mathrm{Y}$ esto último se prueba que es cierto directamente, ya que $(x-y)^{2} \geqslant 0$ siempre.

En estos momentos es ilustrativo reescribir la demostración de forma directa:

$$
\begin{aligned}
& (x-y)^{2} \geqslant 0 \Rightarrow x^{2}+y^{2}-2 x y \geqslant 0 \Rightarrow x^{2}+y^{2}+2 x y \geqslant 4 x y \Rightarrow \\
& (x+y)^{2} \geqslant 4 x y \Rightarrow \sqrt{(x+y)^{2}} \geqslant \sqrt{4 x y} \Rightarrow|x+y| \geqslant 2 \sqrt{x y} \stackrel{x, y>0}{\Rightarrow} \frac{x+y}{2} \geqslant \sqrt{x y}
\end{aligned}
$$




\section{Capítulo 4}

\section{Inducción}

\section{Objetivos}

- Conocer ejemplos concretos de demostraciones por inducción ya sea simple o completa.

- Saber que tanto la inducción simple como la completa tienen dos condiciones que hay que verificar siempre, no siendo correcto comprobar solo una de ellas.

- Ser conscientes de que la inducción completa da más flexibilidad que la inducción simple, aunque teóricamente son equivalentes.

La inducción consiste en inferir una ley general a partir de casos particulares, mientras que la deducción es una inferencia de lo general a lo particular. La deducción es válida siempre que sea aplicada correctamente y aunque a veces la inducción nos dé conclusiones falsas, no se puede despreciar. En general no se puede confiar en el resultado del razonamiento inductivo porque mientras existan casos que no hayan sido considerados, es posible que la regla general inductiva sea incorrecta. Por ejemplo, la desigualdad $n^{2}-3 n-1<0$ es verdadera para $n=1,2,3$ pero tomado $n=4$ no se cumple ya que $4^{2}-3 \cdot 4-1=3>0$. Otro ejemplo sería el dado por el polinomio $P(n)=n^{2}+n+41$ que para los primeros valores naturales proporciona números primos: $P(1)=43, P(2)=47, P(3)=53$, luego se podría pensar que para cualquier número natural se obtendría un número primo pero $P(40)=1681=41^{2}$.

En matemáticas es importante el uso de la inducción pues permite descubrir verdades matemáticas considerando varios casos especiales e infiriendo de ellos una regla general que parece plausible. Sin embargo esa "supuesta " verdad matemática no se puede aceptar basándose en la evidencia inductiva solamente. La diferencia entre la matemática y las ciencias experimentales es que, una vez descubierta por inducción una ley matemática general, se debe demostrar rigurosamente aplicando el proceso deductivo.

Una de las técnicas deductivas más útiles disponibles en matemáticas, tiene la mala fortuna de llamarse inducción matemática.

Es conveniente ver el MDM "Inducción" antes de seguir leyendo este mini-capítulo. 


\subsection{Simple}

Se describe a continuación este tipo particular de deducción llamada inducción matemática simple, que se enuncia como sigue:

Una proposición $p(n)$ relativa a los números naturales es verdadera para todos los valores de la variable $n \in \mathbb{N}$ si se cumplen las dos condiciones siguientes:

1. La proposición $p(k)$ es verdadera para $k=1$, o bien, $p(1)$ es verdadera.

2. Si la proposición $p(k)$ es verdadera, entonces $p(k+1)$ es verdadera, donde $\mathrm{k}$ es un número natural cualquiera.

La primera parte suele ser muy fácil de verificar pero hay que hacerlo (ver el ejemplo del MDM "Inducción"). La segunda parte es la más complicada de comprobar pues se trata de suponer cierta la proposición en un número natural $k$ para deducir que la proposición es también cierta para $k+1$.

El método también se puede aplicar para demostrar la veracidad de $p(n) \forall n \geqslant n_{0}$ comprobando en la primera condición que $p\left(n_{0}\right)$ es verdadera.

\section{Ejemplos}

1. Demostrar que

$$
\forall n \in \mathbb{N} \quad 1+2+\ldots+n=\frac{n(n+1)}{2}
$$

\section{SOLUCIÓN:}

En primer lugar se comprueba que la proposición es verdadera para $k=1$ ya que

$$
1=\frac{1(1+1)}{2}
$$

A continuación se comprueba la segunda condición de la inducción, de manera que si se supone que la igualdad es verdadera para un cierto valor de $n=k$

$$
1+2+\ldots+k=\frac{k(k+1)}{2}
$$

se debe probar que

$$
1+2+\ldots+k+(k+1)=\frac{(k+1)(k+2)}{2}
$$

Esta proposición $p(k+1)$ se obtiene a partir de la hipótesis de inducción $p(k)$ sumando $k+1$ a ambos miembros 
de la igualdad:

$$
1+2+\ldots+k+(k+1)=\frac{k(k+1)}{2}+(k+1)=\frac{k(k+1)+2(k+1)}{2}=\frac{(k+1)(k+2)}{2} .
$$

$\mathrm{Al}$ cumplirse las dos condiciones de inducción simple se puede afirmar que

$$
\forall n \in \mathbb{N} \quad 1+2+\ldots+n=\frac{n(n+1)}{2} .
$$

Obs.: Este resultado ya se probó de otra forma en el ejemplo 1 de la sección 1.2 .

2. Demostrar que

$$
\forall n \in \mathbb{N}, n \geqslant 2 \quad n^{3}-n=\dot{3}
$$

\section{SOLUCIÓN:}

En primer lugar se comprueba que la proposición es verdadera para $k=2$ ya que

$$
2^{3}-2=8-2=6=\dot{3}
$$

A continuación se comprueba la segunda condición de la inducción, de manera que si se supone que la igualdad es verdadera para un cierto valor $k \in \mathbb{N}$, con $k \geqslant 2$; esto es $k^{3}-k=\dot{3}$, se debe probar que $(k+1)^{3}-(k+1)=\dot{3}$.

En efecto

$$
\begin{gathered}
(k+1)^{3}-(k+1)=(k+1)^{2}(k+1)-(k+1) \\
=(k+1)\left[(k+1)^{2}-1\right]=(k+1)\left(k^{2}+2 k\right) \\
=k^{3}+3 k^{2}+2 k \\
=k^{3}-k+3 k^{2}+3 k \stackrel{\text { H.ind. }}{=} \dot{3}+\dot{3}+\dot{3}=\dot{3} .
\end{gathered}
$$

Obs. 1: Otra forma de demostrar la tesis de inducción es razonando sobre $(k+1)^{3}-(k+1)=(k+1)\left[(k+1)^{2}-1\right]=$ $k(k+1)(k+2)$ y al ser tres números consecutivos alguno tiene que ser múltiplo de 3.

Obs. 2: Este resultado también se puede probar de forma directa distinguiendo dos casos $n=2 k$ y $n=2 k+1$.

3. Demostrar que

$$
\forall n \in \mathbb{N} \quad\left(\begin{array}{c}
n \\
0
\end{array}\right)+\left(\begin{array}{c}
n \\
1
\end{array}\right)+\ldots+\left(\begin{array}{l}
n \\
n
\end{array}\right)=2^{n}
$$

\section{SOLUCIÓN:}


La igualdad es cierta para $k=1$ :

$$
\left(\begin{array}{l}
1 \\
0
\end{array}\right)+\left(\begin{array}{l}
1 \\
1
\end{array}\right)=1+1=2^{1}
$$

La hipótesis de inducción es

$$
\left(\begin{array}{l}
k \\
0
\end{array}\right)+\left(\begin{array}{l}
k \\
1
\end{array}\right)+\ldots+\left(\begin{array}{l}
k \\
k
\end{array}\right)=2^{k}
$$

y la tesis

$$
\left(\begin{array}{c}
k+1 \\
0
\end{array}\right)+\left(\begin{array}{c}
k+1 \\
1
\end{array}\right)+\ldots+\left(\begin{array}{l}
k+1 \\
k+1
\end{array}\right)=2^{k+1}
$$

Para comprobar la tesis de inducción, se usa ahora la igualdad

$$
\left(\begin{array}{c}
n \\
m
\end{array}\right)+\left(\begin{array}{c}
n \\
m+1
\end{array}\right)=\left(\begin{array}{c}
n+1 \\
m+1
\end{array}\right)
$$

demostrada de manera directa en la sección 1.2 , con lo que

$$
\begin{gathered}
\left(\begin{array}{c}
k+1 \\
0
\end{array}\right)+\left(\begin{array}{c}
k+1 \\
1
\end{array}\right)+\ldots+\left(\begin{array}{c}
k+1 \\
k
\end{array}\right)+\left(\begin{array}{l}
k+1 \\
k+1
\end{array}\right) \\
=\left(\begin{array}{c}
k+1 \\
0
\end{array}\right)+\left[\left(\begin{array}{l}
k \\
0
\end{array}\right)+\left(\begin{array}{l}
k \\
1
\end{array}\right)\right]+\left[\left(\begin{array}{l}
k \\
1
\end{array}\right)+\left(\begin{array}{l}
k \\
2
\end{array}\right)\right]+\ldots+\left[\left(\begin{array}{c}
k \\
k-1
\end{array}\right)+\left(\begin{array}{l}
k \\
k
\end{array}\right)\right]+\left(\begin{array}{l}
k+1 \\
k+1
\end{array}\right) \\
=\left(\begin{array}{c}
k+1 \\
0
\end{array}\right)+\left(\begin{array}{l}
k \\
0
\end{array}\right)+2\left(\begin{array}{l}
k \\
1
\end{array}\right)+2\left(\begin{array}{l}
k \\
2
\end{array}\right)+\ldots+2\left(\begin{array}{c}
k \\
k-1
\end{array}\right)+\left(\begin{array}{l}
k \\
k
\end{array}\right)+\left(\begin{array}{l}
k+1 \\
k+1
\end{array}\right) \\
=\left(\begin{array}{c}
k \\
0
\end{array}\right)+\left(\begin{array}{l}
k \\
0
\end{array}\right)+2\left(\begin{array}{l}
k \\
1
\end{array}\right)+2\left(\begin{array}{l}
k \\
2
\end{array}\right)+\ldots+2\left(\begin{array}{c}
k \\
k-1
\end{array}\right)+\left(\begin{array}{l}
k \\
k
\end{array}\right)+\left(\begin{array}{l}
k \\
k
\end{array}\right) \\
=2\left(\left(\begin{array}{c}
k \\
0
\end{array}\right)+\left(\begin{array}{l}
k \\
1
\end{array}\right)+\left(\begin{array}{l}
k \\
2
\end{array}\right)+\ldots+\left(\begin{array}{c}
k \\
k-1
\end{array}\right)+\left(\begin{array}{l}
k \\
k
\end{array}\right)\right) \stackrel{\text { H.ind. }}{=} \cdot 2^{k}=2^{k+1} .
\end{gathered}
$$

\section{Ejercicios}

1. Demostrar que

$$
\forall n \in \mathbb{N} \quad 1+3+5+\ldots+(2 n-1)=n^{2} .
$$

\section{SOLUCIÓN:}

Es inmediata la igualdad para $k=1: 1=1^{2}$.

Se comprobará que a partir de

$$
1+3+5+\ldots+(2 k-1)=k^{2}
$$

se puede deducir

$$
1+3+5+\ldots+(2 k-1)+(2(k+1)-1)=(k+1)^{2} .
$$


En efecto, al sumar $2 k+1$ a ambos lados de la hipótesis de inducción

$$
1+3+5+\ldots+(2 k-1)+(2 k+1)=k^{2}+2 k+1=(k+1)^{2} .
$$

2. Demostrar que

$$
\forall n \in \mathbb{N}, n \geqslant 2 \quad n^{3}-n=\dot{6}
$$

\section{SOLUCIÓN:}

La proposición es verdadera si $k=2: 2^{3}-2=8-2=6=\dot{6}$.

Se supone que es cierto $k^{3}-k=\dot{6}$ y se deduce $(k+1)^{3}-(k+1)=\dot{6}$. Para ello se opera con la tesis

$$
\begin{aligned}
(k+1)^{3}-(k+1) & =(k+1)^{2}(k+1)-(k+1) \\
& =(k+1)\left[(k+1)^{2}-1\right]=(k+1)\left(k^{2}+2 k\right) \\
& =k^{3}+3 k^{2}+2 k=k^{3}-k+3 k^{2}+3 k \\
& =k^{3}-k+3 k(k+1) \stackrel{\text { H.ind. }}{=} \cdot 6+3 k(k+1) .
\end{aligned}
$$

Pero tanto si $k$ es par como si $k$ es impar resulta que $k(k+1)=\dot{2}$, por lo que $3 k(k+1)=\dot{6}$ y queda probado $(k+1)^{3}-(k+1)=\dot{6}+\dot{6}=\dot{6}$.

3. Demostrar que

$$
\forall n \in \mathbb{N}, n \geqslant 4 \quad 2^{n}<n !
$$

\section{SOLUCIÓN:}

Se tiene para $k=4$ que $2^{4}=16<4$ ! $=24$.

Suponiendo cierta la desigualdad $2^{k}<k$ ! se deducirá la veracidad de $2^{k+1}<(k+1)$ ! como sigue

$$
2^{k+1}=2^{k} \cdot 2 \stackrel{\text { H.ind. }}{<} k ! \cdot 2<k !(k+1)=(k+1) !
$$

ya que $2<k+1$ al tomarse $k \geqslant 4$.

\subsection{Completa}

Existe otro principio de inducción, aparentemente más general que el enunciado en la sección anterior, que en realidad es equivalente. Se llama habitualmente segundo principio de inducción, o también principio de inducción completa y se enuncia de la siguiente manera: 
Una proposición $p(n)$ relativa a los números naturales es cierta o verdadera para todos los valores de la variable $n \in \mathbb{N}$ si se cumplen las condiciones:

1. La proposición $p(k)$ es verdadera para $k=1$, o bien, $p(1)$ es verdadera.

2. Si las proposiciones $p(1), p(2), \ldots, p(k)$ son verdaderas, entonces $p(k+1)$ es verdadera, donde $k$ es un número natural cualquiera.

$\mathrm{Al}$ igual que en la inducción simple, se puede aplicar para demostrar la veracidad de $p(n) \forall n \geqslant n_{0}$ comprobando en la primera condición que $p\left(n_{0}\right)$ es verdadera y en la segunda condición que si $p\left(n_{0}\right), p\left(n_{0}+1\right), \ldots, p(k)$ son verdaderas entonces $p(k+1)$ es verdadera. Otra forma equivalente para demostrar la veracidad de $p(n) \forall n \geqslant n_{0}$ es verificar que se cumplen las condiciones:

1. Las proposiciones $p\left(n_{0}\right), p\left(n_{0}+1\right), \ldots, p\left(n_{1}\right)$ son verdaderas.

2. Si las proposiciones $p\left(n_{0}\right), p\left(n_{0}+1\right), \ldots, p(k)$ son verdaderas entonces $p(k+1)$ es verdadera, donde $k \geqslant n_{1}$.

Claramente, la inducción completa da más flexibilidad para probar algo que la inducción tradicional porque:

- Se puede utilizar cualquier hipótesis inductiva previa.

- Si algo se puede demostrar mediante inducción simple también se puede demostrar mediante inducción completa.

\section{Ejemplos}

1. Demostrar que todo número natural $n>1$ puede ser escrito como un producto de números primos.

\section{SOLUCIÓN:}

En primer lugar se verifica que la proposición es verdadera para $k=2$.

A continuación se comprueba la segunda condición de la inducción completa, de manera que se considera el natural $k+1$, y se asume por hipótesis inductiva que todo natural $j \in[2, k]$ puede ser escrito como un producto de primos.

Se plantean dos casos:

- $k+1$ es primo: Trivial.

- $k+1$ no es primo: Existen dos naturales $j, \ell \in[2, k]$ tal que $k+1=j \ell$. Por hipótesis inductiva, $j$ y $\ell$ pueden ser escritos como productos de primos. Se concluye que $k+1$ puede ser escrito como producto de primos.

2. Demostrar que todo número natural $n \geqslant 8$ se puede expresar como suma de treses y cincos. 


\section{SOLUCIÓN:}

En este ejemplo se está considerando la proposición $p(n)$ dada por " $n$ es suma de treses y cincos". Para demostrar la veracidad de $p(n) \forall n \geqslant 8$ se usa la segunda versión de la inducción completa verificando:

a) Las proposiciones $p(8), p(9)$, y $p(10)$ son verdaderas, ya que $8=5+3,9=3+3+3$ y $10=5+5$.

b) Si las proposiciones $p(8), p(9), p(10), \ldots, p(k)$ son verdaderas entonces $p(k+1)$ es verdadera, donde $k \geqslant 10$, ya que si $k \geqslant 10 \Rightarrow 8 \leqslant k+1-3<k$ y resulta que la proposición $p(k+1-3)$ es verdadera, de manera que se puede escribir

$$
k+1-3=3+\ldots+3+5+\ldots+5
$$

lo que lleva a la igualdad

$$
k+1=3+3+\ldots+3+5+\ldots+5
$$

que equivale a afirmar que $p(k+1)$ es verdadera.

\section{Ejercicios}

1. Demostrar que todo número natural $n \geqslant 12$ se puede expresar como suma de cuatros y cincos.

\section{SOLUCIÓN:}

Inicialmente se puede observar que la afirmación $p(n)$ dada por " $n$ es suma de cuatros y cincos" es cierta para los valores de $n=12,13,14$ y 15 ya que al ser $12=4+4+4,13=4+4+5,14=4+5+5$ y $15=5+5+5$, son ciertas $p(12), p(13), p(14)$ y $p(15)$.

Por inducción completa:

Suponiendo ciertas $p(12), p(13), \ldots, p(k)$ con $k \geqslant 15$ se comprueba que $p(k+1)$ es cierta.

Dado que $k \geqslant 15$, resulta que $k-3 \geqslant 12$ y por hipótesis inductiva la propiedad es cierta para $k-3$, de manera que se puede escribir

$$
k-3=4+\ldots+4+5+\ldots+5
$$

y agregando un cuatro a ambos lados de la igualdad se tiene

$$
\begin{gathered}
k-3+4=4+4+\ldots 4+5+\ldots+5 \\
k+1=4+\ldots+4+5+\ldots+5
\end{gathered}
$$

que equivale a afirmar que $p(k+1)$ es verdadera. 
2. Se definen los números $a_{n} \in \mathbb{R}$ para $n \in \mathbb{N}$ de la siguiente manera:

$$
\left\{\begin{array}{cc}
a_{n}=a_{n-1}+a_{n-2} & \forall n \geqslant 3 \\
a_{1}=1 & a_{2}=3
\end{array}\right.
$$

Demostrar que $a_{n}<\left(\frac{7}{4}\right)^{n}$.

\section{SOLUCIÓN:}

Se observa que la proposición $p(n)$ dada por "el número $a_{n}$ verifica que $a_{n}<\left(\frac{7}{4}\right)^{n}$ " es verdadera para $n=1$ pues $a_{1}=1<\frac{7}{4}$.

Suponiendo ciertas $p(1), p(2), \ldots, p(k)$ con $k \geqslant 2$, es decir

$$
a_{1}=1<\frac{7}{4}, \quad a_{2}=3<\left(\frac{7}{4}\right)^{2}, \ldots, a_{k-1}<\left(\frac{7}{4}\right)^{k-1}, \quad a_{k}<\left(\frac{7}{4}\right)^{k}
$$

se comprueba que $p(k+1)$ es cierta

$$
\begin{aligned}
a_{k+1} & =a_{k}+a_{k-1}<\left(\frac{7}{4}\right)^{k}+\left(\frac{7}{4}\right)^{k-1} \\
& =\left(\frac{7}{4}\right)^{k-1}\left(\frac{7}{4}+1\right)=\left(\frac{7}{4}\right)^{k-1}\left(\frac{11}{4}\right) \\
& <\left(\frac{7}{4}\right)^{k-1}\left(\frac{7}{4}\right)^{2}=\left(\frac{7}{4}\right)^{k+1} \cdot \square
\end{aligned}
$$




\section{Capítulo 5}

\section{Primer elemento}

\section{Objetivos}

- Conocer el principio de buena ordenación.

- Conocer el principio de descenso infinito.

- Relacionar ambas metodologías.

Los resultados que se pueden demostrar con el principio de buena ordenación y con el método de descenso infinito vendrán referidos a los números naturales. Ambos procedimientos están muy relacionados y son una variación del método de reducción al absurdo aplicado a $\mathbb{N}$.

Es conveniente ver los MDM["Buena ordenación"] y "Descenso infinito" antes de seguir leyendo este mini-capítulo.

\subsection{Principio de buena ordenación}

El principio de buena ordenación es otra importante propiedad de los números naturales que se enuncia como sigue:

Todo subconjunto $C$ no vacío de $\mathbb{N}$ posee elemento mínimo. Es decir:

$$
\emptyset \neq C \subset \mathbb{N} \Rightarrow \exists m \in C: \quad \forall n \in C \quad m \leqslant n .
$$

Es importante hacer notar que este principio no se verifica en $\mathbb{Q}$ o en $\mathbb{R}$, ahora bien, lo que sí se cumple es que de existir el elemento mínimo de un conjunto, éste es necesariamente único y pertenece al conjunto. Se suele denotar al elemento mínimo de un conjunto $C$ por $\min (C)$.

Aunque aparentemente el principio de buena ordenación y el principio de inducción no tienen nada que ver, se puede demostrar que son equivalentes. 


\section{Ejemplos}

1. Demostrar que $\forall n \in \mathbb{N}$ los números de la forma $2^{n}-(-1)^{n}$ son múltiplos de 3 .

\section{SOLUCIÓN:}

La afirmación que hay que probar es equivalente a definir el conjunto de números naturales

$$
C=\left\{n \in \mathbb{N}: 2^{n}-(-1)^{n} \text { no es múltiplo de } 3\right\}
$$

y comprobar que dicho conjunto es vacío. Si $C$ no fuera vacío, por el principio de buena ordenación, $C$ tendría un mínimo $m$ del que se sabe que:

a) $m>1$ ya que si $m$ fuera 1 , al ser $2^{1}-(-1)^{1}=3=\dot{3}, m=1 \notin C$, lo que estaría en contradicción con el hecho de que el elemento mínimo pertenece al conjunto.

b) $m$ es el menor valor natural positivo que garantiza $2^{m}-(-1)^{m}$ no es múltiplo de 3 , luego para un valor más pequeño, por ejemplo $m-1$, se tendrá que $2^{m-1}-(-1)^{m-1}$ es múltiplo de 3 . Pero si $2^{m-1}-(-1)^{m-1}$ es múltiplo de 3 entonces $2^{m}-2(-1)^{m-1}=6 k$, con lo que $2^{m}=6 k+2(-1)^{m-1}$

- $\operatorname{Si}(-1)^{m-1}=-1 \Rightarrow 2^{m}=6 k-2 \Rightarrow 2^{m}-(-1)^{m}=6 k-2-1=3(2 k-1)=\dot{3}$

- $\operatorname{Si}(-1)^{m-1}=1 \Rightarrow 2^{m}=6 k+2 \Rightarrow 2^{m}-(-1)^{m}=6 k+2+1=3(2 k+1)=\dot{3}$

y, en ambos casos, $2^{m}-(-1)^{m}$ es múltiplo de 3 , lo que produce una contradicción.

Luego $C=\emptyset$.

2. Demostrar que

$$
\forall n \in \mathbb{N} \quad 1+2+\ldots+n=\frac{n(n+1)}{2}
$$

\section{SOLUCIÓN:}

La afirmación que hay que probar es equivalente a definir el conjunto de números naturales

$$
C=\left\{n \in \mathbb{N}: \sum_{k=1}^{n} k \neq \frac{n(n+1)}{2}\right\}
$$

y comprobar que dicho conjunto es vacío.

Suponiendo que $C$ es un conjunto no vacío, el principio de buena ordenación asegura que $\exists m=\operatorname{mín}(C)$, del que se sabe que es distinto de 1 pues claramente $1 \notin C$.

Luego $\forall n<m$ se satisface $\sum_{k=1}^{n} k=\frac{n(n+1)}{2}$ pues $m$ era el menor valor natural positivo para el que no se cumplía dicha desigualdad. En particular se verifica para $m-1$ por lo que: $\sum_{k=1}^{m-1} k=\frac{(m-1) m}{2}$. 
Sumando $m$ a ambos lados de la igualdad, resulta

$$
\begin{gathered}
m+\sum_{k=1}^{m-1} k=m+\frac{(m-1) m}{2} \\
\sum_{k=1}^{m} k=\frac{m(m+1)}{2}
\end{gathered}
$$

lo que produce una contradicción con el hecho de que $m \in C$. Luego $C=\emptyset$.

Obs. Este resultado ya se había demostrado en la sección 1.2 y en la sección 4.1. Es interesante observar que en matemáticas no existe una única demostración, pudiendo haber varias.

\section{Ejercicios}

1. Demostrar que existe un primer número natural $n$ que cumple la condición

$$
1^{n}+2^{n}+\ldots+99^{n}<100^{n}
$$

\section{SOLUCIÓN:}

Se define el conjunto

$$
C=\left\{n \in \mathbb{N}: \quad 1^{n}+2^{n}+\ldots+99^{n}<100^{n}\right\}
$$

Por tanto ahora hay que demostrar que $C \neq \emptyset$. Como

$$
1^{n}+2^{n}+\ldots+99^{n}<\underbrace{99^{n}+99^{n}+\ldots+99^{n}}_{99}<99(99)^{n}
$$

bastaría encontrar un número natural $n$ tal que

$$
99(99)^{n}<100^{n}
$$

o equivalentemente

$$
99<\left(\frac{100}{99}\right)^{n}
$$

lo que se verifica para por ejemplo $n=458$. Por tanto $458 \in C$, y así $C$ no es vacío y por el principio de buena ordenación debe tener un elemento mínimo.

2. Si $a \in \mathbb{Z}$ y $b \in \mathbb{N}$, demostrar que existe un único par de enteros $q, r$ tales que

$$
a=q b+r \quad \text { con } \quad 0 \leqslant r<b .
$$




\section{SOLUCIÓN:}

La existencia se demostrará usando el principio de buena ordenación y la unicidad por reducción al absurdo.

a) Existencia:

Se define el conjunto $C=\{a-x b: \quad x \in \mathbb{Z} \quad$ y $\quad a-x b \geqslant 0\}$ y se comprueba que $C \neq \emptyset$, distinguiendo dos casos:

- Caso I) Si $a \geqslant 0, a-0 \cdot b \in C$.

- Caso II) Si $a<0$, como $b \geqslant 1$ se tiene $a-a b=a(1-b) \geqslant 0$ y así $a-a b \in C$.

Luego $C \neq \emptyset$. Por el principio de buena ordenación, el conjunto $C$ tiene mínimo, al que se denotará por $r$ y en consecuencia, al ser $r \in C, \exists q \in \mathbb{Z}$ tal que $r=a-q b$, con lo que

$$
a=q b+r \quad \text { con } \quad 0 \leqslant r .
$$

Además, puesto que $r=\operatorname{mín}(C)$, entonces

$$
r-b=(a-q b)-b=a-(q+1) b<0
$$

y por tanto $r<b$.

Así queda probado que $\exists q, r \in \mathbb{Z}$ tales que $a=q b+r$ con $0 \leqslant r<b$.

b) Unicidad:

Se supone que $a=q b+r=q^{\prime} b+r^{\prime}$ con $0 \leqslant r, r^{\prime} \leqslant b$. Si además se asume, por ejemplo, que $q^{\prime}<q$ entonces se tendría $q^{\prime}+1 \leqslant q$ y así

$$
r=a-q b \leqslant a-\left(q^{\prime}+1\right) b=\left(a-q^{\prime} b\right)-b=r^{\prime}-b<0
$$

que es una contradicción.

En el caso $q<q^{\prime}$, actuando de manera similar, también se llega a una contradicción. Luego $q=q^{\prime}$ y por tanto, $r=a-q b=a^{\prime}-q^{\prime} b=r^{\prime}$.

Obs. Es importante destacar que este ejercicio corresponde al llamado Teorema de la división. Por ejemplo, si se aplica a la división de 25 entre 7 , se obtiene

$$
C=\{\ldots, 25-(-1) \cdot 7,25-0 \cdot 7,25-1 \cdot 7,25-2 \cdot 7,25-3 \cdot 7\}=\{\ldots, 32,25,18,11,4\}
$$

y $r=\operatorname{mín}(C)=25-3 \cdot 7=4$, con lo que $25=3 \cdot 7+4$, es decir la división de 25 entre 7 da un cociente de 3 y un resto de 4. Además, por el teorema, este par de números es único. 


\subsection{Principio de descenso infinito}

El método de demostración por descenso infinito fue creado por el matemático francés Pierre de Fermat y se usa normalmente a la hora de demostrar que una determinada propiedad no es cierta para un subconjunto concreto de los números naturales. El principio de descenso infinito establece que no existe una secuencia infinita decreciente de números naturales, es decir, no se puede encontrar un conjunto infinito de números naturales $\left\{m_{i} \in \mathbb{N}: m_{1}>m_{2}>\ldots\right\}$.

\section{Ejemplos}

1. Demostrar que $\sqrt{2}$ no es un número racional.

\section{SOLUCIÓN:}

En primer lugar se aplica reducción al absurdo suponiendo que $\sqrt{2}$ es un número racional, es decir $\sqrt{2}=\frac{a}{b}$ con $a, b \in \mathbb{N}$ que equivale a la igualdad $a=b \sqrt{2}$. A continuación se aplica el principio de descenso infinito al conjunto $S=\{n \in \mathbb{N}: n \sqrt{2} \in \mathbb{N}\} \neq \emptyset$. Sea $m_{1}=n_{1} \sqrt{2} \in S$, considerando $m_{2}=m_{1}-n_{1}=n_{1} \sqrt{2}-n_{1}$ se tiene que $m_{2} \in \mathbb{N}$ ya que $\sqrt{2}>1$. Además se verifica que $m_{2} \sqrt{2}=2 n_{1}-n_{1} \sqrt{2} \in \mathbb{N}$, ya que $2>\sqrt{2}$, de manera que $m_{2} \in S$ con $m_{1}>m_{2}$. Siguiendo este proceso se estaría construyendo una secuencia infinita de números naturales $m_{1}>m_{2}>\ldots$, lo que entraría en contradicción con el principio de descenso infinito. Esto significa que el supuesto hecho al principio de que $\sqrt{2}$ es un número racional es falso.

2. Demostrar que no existen soluciones naturales no triviales de la ecuación

$$
a^{2}+b^{2}=3 c^{2}
$$

\section{SOLUCIÓN:}

Se empieza negando la afirmación, de manera que se supone que existen números naturales $a, b$ y $c$ verificando que $a^{2}+b^{2}=3 c^{2}$. A continuación, se define el conjunto $S=\left\{n \in \mathbb{N}: n=a+b+c, a, b, c \in \mathbb{N}\right.$ con $\left.a^{2}+b^{2}=3 c^{2}\right\} \neq \emptyset$. Sea $m_{1} \in S: m_{1}=a_{1}+b_{1}+c_{1}$ con $a_{1}^{2}+b_{1}^{2}=3 c_{1}^{2}$. En primer lugar se observa que $a_{1}^{2}+b_{1}^{2}=3$ y por el ejemplo 2 de la sección 3.1 se tiene que $a_{1}=\dot{3}$ y $b_{1}=\dot{3}$, con lo que $\exists a_{2}, b_{2} \in \mathbb{N}: a_{1}=3 a_{2}$ y $b_{1}=3 b_{2}$, verificando que

$$
\left(3 a_{2}\right)^{2}+\left(3 b_{2}\right)^{2}=3 c_{1}^{2} \Rightarrow 3^{2}\left(a_{2}^{2}+b_{2}^{2}\right)=3 c_{1}^{2} \Rightarrow 3\left(a_{2}^{2}+b_{2}^{2}\right)=c_{1}^{2} \Rightarrow c_{1}^{2}=\dot{3}
$$

Ahora por el ejemplo 2 de la sección $2.1 c_{1}=\dot{3}$, es decir, $\exists c_{2} \in \mathbb{N}$ con $c_{1}=3 c_{2}$. Por tanto

$$
3\left(a_{2}^{2}+b_{2}^{2}\right)=\left(3 c_{2}\right)^{2} \Rightarrow 3\left(a_{2}^{2}+b_{2}^{2}\right)=3^{2} c_{2}^{2} \Rightarrow a_{2}^{2}+b_{2}^{2}=3 c_{2}^{2}
$$

Y se ha encontrado una nueva terna $a_{2}, b_{2}, c_{2}$ verificando que $a_{2}^{2}+b_{2}^{2}=3 c_{2}^{2}$ con $a_{1}>a_{2}, b_{1}>b_{2}$ y $c_{1}>c_{2}$, 
por lo que si se define $m_{2}=a_{2}+b_{2}+c_{2}$ se tiene que $m_{2} \in S$ con $m_{1}>m_{2}$. Siguiendo este proceso se estaría construyendo una secuencia infinita de números naturales $m_{1}>m_{2}>\ldots$, lo que entraría en contradicción con el principio de descenso infinito. Esto significa que el supuesto hecho al principio es falso, con lo que no existen $a, b, c \in \mathbb{N}$ tales que $a^{2}+b^{2}=3 c^{2}$.

\section{Ejercicios}

1. Demostrar que $\sqrt{3}$ no es un número racional.

\section{SOLUCIÓN:}

Se supone que $\sqrt{3}$ es un número racional, es decir $\sqrt{3}=\frac{a}{b}$ con $a, b \in \mathbb{N}$ que equivale a la igualdad $a=b \sqrt{3}$.

Se define el conjunto $S=\{n \in \mathbb{N}: n \sqrt{3} \in \mathbb{N}\} \neq \emptyset$. Sea $m_{1}=n_{1} \sqrt{3} \in S$, considerando $m_{2}=m_{1}-n_{1}=n_{1} \sqrt{3}-n_{1}$ se tiene que $m_{2} \in \mathbb{N}$ ya que $\sqrt{3}>1$. Además se verifica que $m_{2} \sqrt{3}=3 n_{1}-n_{1} \sqrt{3} \in \mathbb{N}$, ya que $3>\sqrt{3}$, de manera que $m_{2} \in S$ con $m_{1}>m_{2}$. Siguiendo este proceso se estaría construyendo una secuencia infinita de números naturales $m_{1}>m_{2}>\ldots$, lo que entraría en contradicción con el principio de descenso infinito. Esto significa que el supuesto hecho al principio de que $\sqrt{3}$ es un número racional es falso.

2. Demostrar que si $x, y \in \mathbb{N}$ tal que $x^{2}+y^{2}+x y$ es divisible por 10 entonces $x$ e $y$ son divisibles por 10 (ver [3] y [4).

\section{SOLUCIÓN:}

Se empieza negando la afirmación y se supone que existen números naturales $x, y$ con $x^{2}+y^{2}+x y=\dot{10}$ siendo $x \neq \dot{10}$ o $y \neq \dot{10}$. A continuación, se define el conjunto $S=\left\{n \in \mathbb{N}: n=x^{2}+y^{2}+x y=\dot{10}\right.$ con $x \neq 1 \dot{0}$ o $y \neq \dot{10}\} \neq \emptyset$. Sea $m_{1} \in S: m_{1}=x^{2}+y^{2}+x y=\dot{10}$ con $x=10 a+k_{1} \quad y=10 b+k_{2}$ siendo $k_{1}, k_{2} \in \mathbb{N}$ con $k_{1} \neq 0$ o $k_{2} \neq 0$. Además $k_{1} \neq \dot{10}$ o $k_{2} \neq \dot{0} 0$ ya que si no $x$ o $y$ serían múltiplos de 10 . Entonces:

$$
\begin{aligned}
x^{2}+y^{2}+x y & =\left(10 a+k_{1}\right)^{2}+\left(10 b+k_{2}\right)^{2}+\left(10 a+k_{1}\right)\left(10 b+k_{2}\right) \\
& =100\left(a^{2}+b^{2}+a b\right)+10\left(2 a k_{1}+2 b k_{2}+a k_{2}+b k_{1}\right)+\left(k_{1}^{2}+k_{2}^{2}+k_{1} k_{2}\right) \\
& =\dot{10}+\left(k_{1}^{2}+k_{2}^{2}+k_{1} k_{2}\right) .
\end{aligned}
$$

$\mathrm{Al}$ ser $x^{2}+y^{2}+x y=\dot{0} 0$ y a la vez $x^{2}+y^{2}+x y=\dot{10}+\left(k_{1}^{2}+k_{2}^{2}+k_{1} k_{2}\right)$, se tiene que $k_{1}^{2}+k_{2}^{2}+k_{1} k_{2}=\dot{10}$ con $k_{1}<x, k_{2}<y$ y $k_{1}^{2}+k_{2}^{2}+k_{1} k_{2} \neq 0$. Con lo que si se define $m_{2}=k_{1}^{2}+k_{2}^{2}+k_{1} k_{2}$, se verifica que $m_{2} \in \mathbb{N}$ con $m_{2}=\dot{10}, k_{1} \neq \dot{10}$ o $k_{2} \neq \dot{10}$, es decir, $m_{2} \in S$ con $m_{1}>m_{2}$. Siguiendo este proceso se estaría construyendo una secuencia infinita de números naturales $m_{1}>m_{2}>\ldots$, lo que entraría en contradicción con el principio de descenso infinito. Esto significa que el supuesto hecho al principio es falso, con lo que si $x, y \in \mathbb{N}$ tal que $x^{2}+y^{2}+x y$ es divisible por 10 entonces $x$ e $y$ son divisibles por 10 . 


\section{Capítulo 6}

\section{Autoevaluación de contenidos}

\subsection{Directas y contras}

\section{Nivel 1}

1. Sean $k_{1}, k_{2} \in \mathbb{N}$, entonces $\frac{k_{1}}{k_{2}} \in \mathbb{N}$.
a) Verdadero.
b) Falso.

2. Sea $k \in \mathbb{N}$, entonces $\frac{k(k+1)}{2} \in \mathbb{N}$.
a) Verdadero.
b) Falso.

3. Sean $k_{1}, k_{2} \in \mathbb{N}$, entonces $\sqrt{k_{1}+k_{2}}=\sqrt{k_{1}}+\sqrt{k_{2}}$.
a) Verdadero.
b) Falso.

4. Sea $k \in \mathbb{N}$, entonces $\left(\begin{array}{l}k \\ 0\end{array}\right)=1$.
a) Verdadero.
b) Falso.

5. Sea $n \in \mathbb{N}$, entonces $1+2+3+\ldots+n=n^{2}$.
a) Verdadero. 
b) Falso.

6. Sea $k \in \mathbb{N}$, entonces $|k|^{2}=k^{2}$.

a) Verdadero.

b) Falso.

\section{Nivel 2}

1. Sean $k_{1}, k_{2} \in \mathbb{N}$, entonces se verifica que:
a) $\frac{k_{1} k_{2}}{2} \in \mathbb{N}$.
b) $\frac{k_{1}^{2}\left(k_{1}^{2}+1\right)}{2} \in \mathbb{N}$.
c) $\frac{k_{1}+k_{2}}{2} \in \mathbb{N}$.
d) $\frac{k_{1}^{2}\left(k_{2}+1\right)}{3} \in \mathbb{N}$.

2. Sean $k_{1}, k_{2} \in \mathbb{N}$, entonces se verifica que:
a) $k_{1} k_{2}=\dot{3} \Rightarrow k_{1}=\dot{3}$.
b) $k_{1} k_{2}=\dot{4} \Rightarrow k_{1}=\dot{4}$ y $k_{2}=\dot{4}$.
c) $k_{1} k_{2}=\dot{6} \Rightarrow k_{1}=\dot{3}$ y $k_{2}=\dot{2}$.
d) $k_{1} k_{2}=\dot{4} \Rightarrow k_{1}=\dot{2}$ о $k_{2}=\dot{2}$.

3. Sean $k_{1}, k_{2} \in \mathbb{N}$, entonces se verifica que:
a) $\sqrt{k_{1}\left(k_{2}+1\right)}=\sqrt{k_{1}}+\sqrt{k_{2}+1}$.
b) $\sqrt{k_{1}\left(k_{2}+1\right)}=\sqrt{k_{1} k_{2}}+\sqrt{k_{1}}$.
c) $\sqrt{k_{1}\left(k_{2}+1\right)}=\sqrt{k_{1}} \sqrt{k_{2}}+\sqrt{k_{1}}$.
d) $\sqrt{k_{1}\left(k_{2}+1\right)}=\sqrt{k_{1}} \sqrt{k_{2}+1}$.

4. Sean $k_{1}, k_{2} \in \mathbb{N}$, entonces se verifica que:
a) $\left(\begin{array}{c}k_{1} \\ 0\end{array}\right)+\left(\begin{array}{c}k_{2} \\ 0\end{array}\right)=k_{1}+k_{2}$.
b) $\left(\begin{array}{c}k_{1} \\ 1\end{array}\right)+\left(\begin{array}{c}k_{2} \\ 1\end{array}\right)=k_{1}+k_{2}$.
c) $\left(\begin{array}{c}k_{1} \\ 0\end{array}\right)+\left(\begin{array}{c}k_{2} \\ 0\end{array}\right)=k_{1} k_{2}$.
d) $\left(\begin{array}{c}k_{1} \\ 1\end{array}\right)\left(\begin{array}{c}k_{2} \\ 0\end{array}\right)=\left(\begin{array}{c}k_{1} \\ 0\end{array}\right)\left(\begin{array}{c}k_{2} \\ 1\end{array}\right)$. 
5. Sea $n \in \mathbb{N}$, entonces se verifica que $(3 n)^{3}-3 n$ es múltiplo de:
a) $3^{3}$
b) 3 .
c) $3^{2}$
d) 2 .

6. Sean $k_{1}, k_{2} \in \mathbb{N}$, entonces se verifica que:
a) $\left|k_{1}-k_{2}\right|=\left|k_{1}\right|-\left|k_{2}\right|$.
b) $k_{1}\left(-k_{2}\right)=\left|k_{1}\right|\left|-k_{2}\right|$.
c) $\left|k_{1} k_{2}\right|^{2}=\left|k_{1}\right|^{2}\left|k_{2}\right|^{2}$.
d) $\left|k_{1}\right|-\left|k_{2}\right|=k_{2}-k_{1}$.

\subsection{Necesaria y suficiente}

\section{Nivel 1}

1. Se supone que $A \Rightarrow B$ y que $B \nRightarrow A$, entonces se puede decir que no $B$ es condición necesaria para no $A$.
a) Verdadero.
b) Falso.

2. Se supone que $A \Rightarrow B$ y que $B \nRightarrow A$, entonces se puede decir que no $B$ es condición suficiente para no $A$.
a) Verdadero.
b) Falso.

3. Se supone que $A \Rightarrow B$ y que $B \nRightarrow A$, entonces se puede decir que no $A$ es condición necesaria para no $B$.
a) Verdadero.
b) Falso.

4. Se supone que $A \Leftrightarrow B$, entonces se puede decir que no $A$ es condición necesaria y suficiente para $B$.
a) Verdadero.
b) Falso.

5. Se supone que $A \Leftrightarrow B$, entonces se puede decir que $A$ es condición necesaria pero no suficiente para $B$. 
a) Verdadero.

b) Falso.

6. Se supone que $A \Leftrightarrow B$, entonces se puede decir que no $A$ es condición necesaria y suficiente para no $B$.

a) Verdadero.

b) Falso.

\section{Nivel 2}

1. Se sabe que es cierto que si $p>3$ se verifica que $p$ primo $\Rightarrow p^{2}-1=\dot{12}$ y también que $p^{2}-1=\dot{12} \nRightarrow p$ primo, entonces:

a) Es necesario que $p^{2}-1=\dot{12}$ para que $p$ sea primo.

b) Es suficiente que $p^{2}-1=\dot{12}$ para que $p$ sea primo.

c) No es necesario que $p^{2}-1=\dot{12}$ para poder decir que $p$ es primo.

d) No es suficiente que $p$ sea primo para poder decir que $p^{2}-1=\dot{12}$.

2. Se sabe que es cierto que si $p>3$ se verifica que $p$ primo $\Rightarrow p^{2}-1=\dot{12}$ y también que $p^{2}-1=\dot{12} \nRightarrow p$ primo, entonces:

a) Es suficiente que $p^{2}-1 \neq \dot{1} 2$ para que $p$ sea primo.

b) Es necesario que $p^{2}-1 \neq \dot{12}$ para poder decir que $p$ es primo.

c) Es suficiente que $p^{2}-1 \neq \dot{12}$ para poder decir que $p$ es compuesto.

d) Es necesario que $p^{2}-1 \neq \dot{12}$ para poder decir que $p$ es compuesto.

3. Se sabe que es cierto que si $p>3$ se verifica que $p$ primo $\Rightarrow p^{2}-1=\dot{12}$ y también que $p^{2}-1=\dot{12} \nRightarrow p$ primo. Se calcula $p^{2}-1$.
a) Si $p^{2}-1=\dot{12}$ entonces $p$ es compuesto.
b) Si $p^{2}-1=\dot{12}$ entonces $p$ es primo.
c) Si $p^{2}-1 \neq \dot{12}$ entonces no se sabe si $p$ es primo o compuesto.
d) Si $p^{2}-1 \neq \dot{12}$ entonces $p$ es compuesto.

4. Se sabe que es cierto que $p$ primo $\Leftrightarrow(p-1) !+1=\dot{p}$, entonces:

a) Es necesario que $(p-1) !+1=\dot{p}$ para que $p$ sea primo. 
b) Es suficiente que $(p-1) !+1 \neq \dot{p}$ para que $p$ sea primo.

c) No es suficiente que $(p-1) !+1=\stackrel{\bullet}{p}$ para poder decir que $p$ es primo.

d) Es necesario y suficiente que $(p-1) !+1 \neq \dot{p}$ para que $p$ sea primo.

5. Se sabe que es cierto que $p$ primo $\Leftrightarrow(p-1) !+1=\dot{p}$, entonces:

a) Es suficiente que $(p-1) !+1=\dot{p}$ para poder decir que $p$ es primo.

b) Es suficiente que $(p-1) !+1=\stackrel{\bullet}{p}$ para que $p$ sea compuesto.

c) Es necesario que $(p-1) !+1 \neq \dot{p}$ para poder decir que $p$ es primo.

d) Es necesario y suficiente que $(p-1) !+1 \neq \dot{p}$ para que $p$ sea impar.

6. Se sabe que es cierto que $p$ primo $\Leftrightarrow(p-1) !+1=\stackrel{\bullet}{p}$. Se calcula $(p-1) !+1$.

a) Si $(p-1) !+1$ es múltiplo de $p$ entonces no se sabe si $p$ es primo o compuesto.

b) Si $(p-1) !+1$ no es múltiplo de $p$ entonces no se sabe si $p$ es primo o compuesto.

c) Si $(p-1) !+1$ es múltiplo de $p$ entonces $p$ es primo.

d) $\mathrm{Si}(p-1) !+1$ no es múltiplo de $p$ entonces $p$ es primo.

\subsection{Supongamos que}

\section{Nivel 1}

1. Se quiere demostrar que $A \Rightarrow B$ utilizando el método de reducción al absurdo. Para ello hay que suponer que no es cierto $A$ e intentar llegar a una contradicción.
a) Verdadero.
b) Falso.

2. Se quiere demostrar que $A \Rightarrow B$ utilizando el método de reducción al absurdo. Para ello hay que suponer que no $B \Rightarrow$ no $A$.
a) Verdadero.
b) Falso.

3. Se quiere demostrar que $A \Rightarrow B$ utilizando el método de reducción al absurdo. Para ello hay que encontrar una condición $C$ más sencilla de forma que $A \Rightarrow C \Rightarrow B$.

a) Verdadero. 
b) Falso.

4. Se quiere demostrar que $A \Rightarrow B$ utilizando el método de "marcha hacia atrás". Para ello hay que suponer que no $B$ junto con $A$ lleva a una contradicción.

a) Verdadero.

b) Falso.

5. Se quiere demostrar que $A \Rightarrow B$ utilizando el método de "marcha hacia atrás". Para ello hay que encontrar una condición $C$ de tal forma que $A \Rightarrow C$ y $B \Rightarrow C$.

a) Verdadero.

b) Falso.

6. Se quiere demostrar que $A \Rightarrow B$ utilizando el método de "marcha hacia atrás". Para ello una posibilidad es encontrar una condición $C$ de tal forma que $A \Rightarrow C \Leftrightarrow B$.

a) Verdadero.

b) Falso.

\section{Nivel 2}

1. Si $n=4$, entonces se verifica que:
a) $\sqrt{n} \notin \mathbb{Q}$.
b) $\sqrt[3]{n} \notin \mathbb{Q}$.
c) $\sqrt{n} \sqrt{n} \notin \mathbb{Q}$.
d) $\left(\frac{\sqrt[3]{n}}{\sqrt[3]{n^{2}}}\right)^{3} \notin \mathbb{Q}$.

2. Si $n=6$, entonces se verifica que:
a) $(\sqrt[3]{n})^{9} \notin \mathbb{Q}$.
b) $\sqrt[3]{n^{4}} \notin \mathbb{Q}$.
c) $\sqrt[3]{n} \sqrt[3]{n} \sqrt[3]{n} \notin \mathbb{Q}$.
d) $\left(\frac{\sqrt[3]{n^{4}}}{\left(\sqrt[3]{n^{2}}\right)^{2}}\right)^{3} \notin \mathbb{Q}$.

3. Se cumple que: 

a) $\sqrt{6}+\sqrt{2}>\sqrt{15}$.
b) $\sqrt{6}-\sqrt{2}>1$.
c) $\sqrt{7}+\sqrt{3}<\sqrt{17}$.
d) $\sqrt{7}-\sqrt{3}<1$.

4. Sean $0<x<1,0<y<1$, entonces:
a) $0<x+y<1$.
b) $0<x y-x<1$.
c) $0<x-y-x y<1$.
d) $0<x+y-x y<1$.

5. Sean $0<a<x<b$, entonces:
a) $\frac{1}{x}+\frac{1}{a+b-x}<\frac{1}{a}+\frac{1}{b}$.
b) $\frac{1}{x}+\frac{1}{a+b-x}>\frac{1}{a}+\frac{1}{b}$.
c) $\frac{1}{x} \cdot \frac{1}{a+b-x}<\frac{1}{b}-\frac{1}{a}$.
d) $\frac{1}{x} \cdot \frac{1}{a+b-x}<a-b$.

6. Sean $a, b>0$, entonces:
a) $\frac{a}{2(a+b) \sqrt{b}}>\frac{1}{\sqrt{b}}-\frac{1}{\sqrt{a+b}}$.
b) $\frac{a}{2(a+b) \sqrt{b}}<\frac{1}{\sqrt{b}}-\frac{1}{\sqrt{a+b}}$.
c) $\frac{a}{2(a+a) \sqrt{a}}>\frac{1}{\sqrt{a}}-\frac{1}{\sqrt{a+a}}$.
d) $\frac{a}{2(a+b) \sqrt{b}}<\frac{1}{\sqrt{a+b}}-\frac{1}{\sqrt{b}}$.

\subsection{Inducción}

\section{Nivel 1}

1. El principio de inducción debería llamarse de deducción.
a) Verdadero.
b) Falso.

2. Sea $k \in \mathbb{N}$. Si $p(k)$ cierta $\Rightarrow p(k+1)$ cierta, entonces la proposición $p$ es cierta para todo $k$. 

a) Verdadero.
b) Falso.

3. Si $p(1)$ cierta entonces $p(2)$ es cierta por el principio de inducción simple.
a) Verdadero.
b) Falso.

4. Si se demuestra que $p(k), p(k+1), p(k+2)$ son ciertas $\Rightarrow p(k+3)$ es cierta, entonces la proposición $p$ es cierta $\forall k \in \mathbb{N}$, por el principio de inducción completa.
a) Verdadero.
b) Falso.

5. Si $p(1), p(2), \ldots, p\left(10^{6}\right)$ son ciertas entonces $p(k)$ cierta $\forall k \in \mathbb{N}$, por el principio de inducción completa.
a) Verdadero.
b) Falso.

6. Principio de inducción simple $\Rightarrow$ Principio de inducción completa, pero Principio de inducción completa $\nRightarrow$ Principio de inducción simple.
a) Verdadero.
b) Falso.

\section{Nivel 2}

1. Si $n \in \mathbb{N}$, entonces se verifica que $3+6+9+\ldots+3 n$ es igual a:
a) $\frac{3 n(n+1)}{2}$.
b) $\frac{n(n+1)}{2}$.
c) $\frac{3 n(n+3)}{2}$.
d) $\frac{3 n(3 n+1)}{2}$.

2. Si $n \in \mathbb{N}$, entonces se verifica que $n^{2}-n$ es múltiplo de:
a) 3 .
b) 2 . 

c) 5 .
d) 6 .

3. Si $n \in \mathbb{N}$, entonces se verifica que $\left(\begin{array}{c}n-1 \\ 0\end{array}\right)+\left(\begin{array}{c}n-1 \\ 1\end{array}\right)+\ldots+\left(\begin{array}{c}n-1 \\ n-1\end{array}\right)$ es igual a:
a) $2^{n}$
b) $2^{n-1}$.
c) $\frac{(n-1)+n}{2}$.
d) 1 .

4. Si $n \in \mathbb{N}$, entonces se verifica que $2+6+10+\ldots+(4 n-2)$ es igual a:
a) $\frac{n(n+1)}{2}$.
b) $n^{2}$.
c) $4 n^{2}$
d) $2 n^{2}$.

5. Si $n \in \mathbb{N}$, entonces se verifica que $4+5+6+\ldots+(n+3)$ es igual a:
a) $\frac{n(n+1)+6}{2}$.
b) $\frac{n(n+1)}{6}+3$.
c) $n^{2}+3$.
d) $\frac{n(n+7)}{2}$.

6. Si $n \in \mathbb{N}$, entonces se verifica que $4+6+8+\ldots+(2 n+2)$ es igual a:
a) $n(n+1)$.
b) $\frac{n(n+1)}{2}$.
c) $n^{2}+3 n$.
d) $n^{2}+3$

\subsection{Primer elemento}

\section{Nivel 1}

1. El principio de buena ordenación afirma que todo conjunto no vacío de números naturales tiene máximo. 

a) Verdadero.
b) Falso.

2. El principio de buena ordenación afirma que todo conjunto tiene un elemento mínimo.
a) Verdadero.
b) Falso.

3. Si un conjunto $A \subseteq \mathbb{N}$ no tiene mínimo $\Rightarrow A=\emptyset$.
a) Verdadero.
b) Falso.

4. El principio de descenso infinito se basa en el principio de buena ordenación.
a) Verdadero.
b) Falso.

5. El principio de inducción se puede considerar como un proceso de descenso infinito.
a) Verdadero.
b) Falso.

6. El principio de descenso infinito se basa en que si $A$ es un subconjunto de $\mathbb{N}: \forall n \in A, \exists m \in A$ con $m<n \Rightarrow$ $A=\emptyset$.
a) Verdadero.
b) Falso.

\section{Nivel 2}

1. El conjunto $C=\left\{m \in \mathbb{N}: \sum_{k=1}^{m} k \neq \frac{m(m+1)}{2}\right\}$ verifica que:
a) $k \in C$.
b) $1 \in C$.
c) $0 \in C$.
d) $C=\emptyset$.

2. El conjunto $C=\left\{m \in \mathbb{N}: 1000=2^{s} m\right.$ para algún $\left.s \in \mathbb{N}\right\}$ verifica que: 

a) $s \in C$.
b) $1 \in C$.
c) $0 \in C$.
d) $C \neq \emptyset$.

3. El conjunto $C=\left\{m \in \mathbb{N}: 2^{m}-(-1)^{m}\right.$ es múltiplo de 5$\}$ verifica que:
a) $1 \in C$.
b) $3 \in C$.
c) $5 \in C$.
d) $C=\emptyset$.

4. Si existieran $n, a, b \in \mathbb{N}$ verificando $7^{n}=a^{3}+b^{3}$ se tendría:
a) $7^{n-3}=\left(\frac{a}{7}\right)^{3}+\left(\frac{b}{7}\right)^{3}$
b) $7^{n}=\left(\frac{a}{7}\right)^{3}+\left(\frac{b}{7}\right)^{3}$.
c) $7^{n-3}=a^{3-3}+b^{3-3}$.
d) $7^{n+3}=\left(\frac{a}{7}\right)^{3}+\left(\frac{b}{7}\right)^{3}$.

5. Si existieran $a, b, c \in \mathbb{N}$ verificando que $a^{3}+2 b^{3}=4 c^{3}$ se tendría:
a) a impar.
b) $a$ par y $b$ impar.
c) a par y $c$ impar.
d) $a$ par, $b$ par y $c$ par.

6. Si existieran $a, b \in \mathbb{N}$ verificando que $\sqrt{2}=\frac{a}{b}$ se tendría:
a) $\frac{2 b+a}{a-b}=\sqrt{2}$.
b) $\frac{2 b-a}{a-b}=\sqrt{2}$.
c) $\frac{2 b+a}{a-b}=\sqrt{2}$.
d) $\frac{2 b-a}{a+b}=\sqrt{2}$. 


\subsection{Respuestas correctas}

\begin{tabular}{|c|c|c|c|c|c|c|}
\hline & 1 & 2 & 3 & 4 & 5 & 6 \\
\hline 6.1 .1 & $\mathrm{~b}$ & $\mathrm{a}$ & $\mathrm{b}$ & $\mathrm{a}$ & $\mathrm{b}$ & $\mathrm{a}$ \\
6.1 .2 & $\mathrm{~b}$ & $\mathrm{~d}$ & $\mathrm{~d}$ & $\mathrm{~b}$ & $\mathrm{~b}$ & $\mathrm{c}$ \\
\hline 6.2 .1 & $\mathrm{~b}$ & $\mathrm{a}$ & $\mathrm{a}$ & $\mathrm{b}$ & $\mathrm{b}$ & $\mathrm{a}$ \\
6.2 .2 & $\mathrm{a}$ & $\mathrm{c}$ & $\mathrm{d}$ & $\mathrm{a}$ & $\mathrm{a}$ & $\mathrm{c}$ \\
\hline 6.3 .1 & $\mathrm{~b}$ & $\mathrm{~b}$ & $\mathrm{~b}$ & $\mathrm{~b}$ & $\mathrm{~b}$ & $\mathrm{a}$ \\
6.3 .2 & $\mathrm{~b}$ & $\mathrm{~b}$ & $\mathrm{~b}$ & $\mathrm{~d}$ & $\mathrm{a}$ & $\mathrm{b}$ \\
\hline 6.4 .1 & $\mathrm{a}$ & $\mathrm{b}$ & $\mathrm{b}$ & $\mathrm{b}$ & $\mathrm{b}$ & $\mathrm{b}$ \\
6.4 .2 & $\mathrm{a}$ & $\mathrm{b}$ & $\mathrm{b}$ & $\mathrm{d}$ & $\mathrm{d}$ & $\mathrm{c}$ \\
\hline 6.5 .1 & $\mathrm{~b}$ & $\mathrm{~b}$ & $\mathrm{a}$ & $\mathrm{a}$ & $\mathrm{b}$ & $\mathrm{a}$ \\
6.5 .2 & $\mathrm{~d}$ & $\mathrm{~d}$ & $\mathrm{~d}$ & $\mathrm{a}$ & $\mathrm{d}$ & $\mathrm{b}$ \\
\hline
\end{tabular}

Cuadro 6.1: Respuestas correctas 


\section{Capítulo 7}

\section{Glosario}

- Conjunto: Colección de elementos. Los elementos de un conjunto se escriben entre llaves y usualmente con letras minúsculas latinas. Por otra parte, a los conjuntos se les suele denotar por letras mayúsculas latinas.

- Conjunto vacío: El conjunto vacío representa un conjunto sin elementos. Se denota por $\emptyset$. Por ejemplo el conjunto de números primos pares mayores que 3 es el conjunto vacío.

- Contenido: Un conjunto $A$ se dice contenido en otro $B$, y se denota por $A \subset B$, si todo elemento de $A$ está en B. Por ejemplo $\mathbb{N} \subset \mathbb{Z}$.

- Cuadrado perfecto: Un número natural $n$ es cuadrado perfecto, si existe un número natural $a$ tal que $a^{2}=n$.

- Desigualdades: Una expresión de la forma $a \leq b$ representa que $a$ es menor o igual que $b$, o dicho de otra forma que $b-a \geq 0$, es decir que $b-a$ es mayor o igual que cero. Si se verifica que $a<b$ y $c>0$ se tiene que $a c<b c$, pero si $a<b$ y $c<0$ se tiene que $a c>b c$.

- División por cero: ¡iNo se puede dividir por cero!! No existe, por ejemplo $\frac{4}{0}$.

- Divisor: Un número entero $a$ distinto de cero es un divisor de un número entero $b$ si la división de $b$ entre $a$ es exacta.

- Existe: El símbolo para el cuantificador "existe" es $\exists$.

- Factorial: El factorial del número natural $n$ se denota por $n$ ! y está definido por $n$ ! $=n(n-1)(n-2) \ldots 2 \cdot 1$, teniendo en cuenta que $0 !=1$.

- Igualdades básicas algebraicas: "Cuadrado de una suma": $(a+b)^{2}=a^{2}+2 a b+b^{2}$, "cuadrado de una diferencia": $(a-b)^{2}=a^{2}-2 a b+b^{2}$, suma por diferencia o diferencia de cuadrados: $(a+b)(a-b)=a^{2}-b^{2}$. 
- Múltiplo: Un número entero $b$ es un múltiplo de un número entero $a$ distinto de cero, si la división de $b$ entre $a$ es exacta. Se suele escribir $b=\dot{a}$.

- Multiplicación con signos: Esta propiedad afirma que "más por más" es más, "menos por más" es menos, "más por menos" es menos y "menos por menos" es más.

- Números compuestos: Los números naturales que no son primos, son compuestos. El 1 no es ni primo ni compuesto, es la unidad.

- Números enteros: El conjunto de los números enteros está dado por

$$
\{\ldots,-4,-3,-2,-1,0,1,2,3,4, \ldots\} .
$$

Se le denota por $\mathbb{Z}$.

- Números impares: Aquellos números enteros que al dividirlos por dos, no proporcionan un resultado exacto. El conjunto de los números impares es, por tanto, $\{\ldots,-3,-1,1,3, \ldots\}$.

- Números naturales: El conjunto de los números naturales está dado por

$$
\{1,2,3,4, \ldots\} \text {. }
$$

Se le denota por $\mathbb{N}$.

- Números pares: Aquellos números enteros que al dividirlos por dos, proporcionan un resultado exacto. El conjunto de los números pares es, por tanto, $\{\ldots,-4,-2,0,2,4, \ldots\}$. ¡Ojo, que el cero es par!, aunque haya personas que piensan que el cero es comodín y que vale como par o impar.

- Números primos: Un número natural es primo si solo tiene por divisores al 1 y a él mismo.

- Números racionales: El conjunto de los números racionales está dado por

$$
\left\{\frac{n}{m}, n \in \mathbb{Z}, m \in \mathbb{Z}-\{0\}\right\}
$$

Se le denota por $\mathbb{Q}$.

- Números reales: Los números reales, de forma intuitiva, están constituidos por todos los puntos de la recta del eje horizontal de coordenadas. Se le denota por $\mathbb{R}$.

- Para todo: El símbolo para el cuantificador "para todo" es $\forall$. 
- Pertenece: Un elemento $a$ se dice que pertenece a un conjunto $A$, y se denota por $a \in A$, si $a$ se encuentra en A. Por ejemplo, $0 \in \mathbb{Z}$.

- Potencia: La potencia $a^{b}$ tiene por base $a$ y exponente $b$. En el caso de que $b \in \mathbb{N}$, se define $a^{b}=\overbrace{a \cdot \ldots \cdot a}^{b \text { veces }}$. En el caso de que $b=0$, se define $a^{0}=1$. Si $b \in \mathbb{Z}$ y $b=-m<0, a^{b}=\frac{1}{a^{m}}$. Por último si $b \in \mathbb{Q}$ y $b=\frac{m}{n}, a^{b}=\sqrt[n]{a^{m}}$.

- Raíz cuadrada: La raíz cuadrada $\sqrt{a}$, con $a$ positivo es un número $b$ positivo tal que la potencia $b^{2}=a$. Se verifica que $\sqrt{a b}=\sqrt{a} \sqrt{b}$. Evidentemente no es cierto $\sqrt{a+b}=\sqrt{a}+\sqrt{b}$. Por otra parte, si se quiere resolver por ejemplo la ecuación $b^{2}=16$ se tendría $b= \pm \sqrt{16}= \pm 4$.

- Raíz cúbica: La raíz cúbica de un número $a$ es un número $b$ tal que la potencia cúbica de $b$ es $a$. Se denota por $\sqrt[3]{a}=b$

- Sumatorio: El símbolo de sumatorio es la letra $\sum$ que indica de forma abreviada una suma. Por ejemplo, $\sum_{i=1}^{n} i=1+2+\ldots+(n-1)+n$. 


\section{Bibliografía}

[1] De Guzmán, M., Aventuras matemáticas, Labor, 1986.

[2] Dunham, W., El universo de las matemáticas: un recorrido alfabético por los grandes teoremas, enigmas y controversias, Pirámide, 1995.

[3] Engel, A., Problem-solving strategies, Springer, 1998.

[4] Kasiri, H., Shahidikia, A. (2015) A Forgotten Idea! Infinite Descent as a Problem-Solving Heuristic. International Journal of Scientific Engineering and Applied Science (IJSEAS) 1(8) 363-367. 


\section{Acerca de los autores}

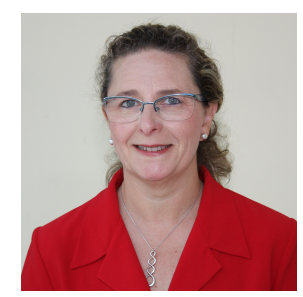

Ma Ángeles Rincón nace en Madrid en 1966. Es Licenciada en Matemáticas por la UCM en 1989. En la actualidad es profesora colaboradora de la UPM en el departamento de Matemática Aplicada a la Ingeniería Industrial. Anteriormente fue profesora de enseñanzas medias. Entre sus líneas de investigación se encuentran modelos estocásticos para poblaciones. Ha participado en tres proyectos de innovación docente de innovación docente en los últimos años siendo coordinadora en dos de ellos y pertenece a un grupo de innovación educativa que colabora con la UNED.

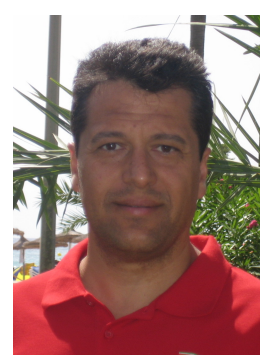

Emilio Letón nace en Madrid en 1966. Es Licenciado en Matemáticas por la UCM en 1989 y doctor en Matemáticas en la misma universidad en 2002. En la actualidad es profesor contratado doctor de la UNED en el departamento de Inteligencia Artificial, al que se incorporó en 2009. Anteriormente fue profesor del departamento de Estadística de la UC3M durante 5 años. Asimismo, ha trabajado durante 15 años en departamentos de Planificación y Estadística dentro del sector bancario y de la industria farmacéutica. Sus líneas de investigación incluyen el Análisis de Supervivencia, tests no paramétricos, PLS, Meta-Análisis, Bioestadística y B-Learning. Además, es Coordinador del grupo miniXmodular. Más información en www.ia.uned.es/ eleton 